\title{
El-Zuma Burials: Analysis of Human Skeletal Remains
}

\author{
Robert Mahler with appendix by Abagail Breidenstein
}

Introduction

The aim of the osteological analysis of human remains recovered from the tumuli at El-Zuma was to reconstruct the demographic and physical health profile of the buried individuals by collecting the best dataset possible on bone morphology. The relatively small number of tombs recorded (29 in total) and the overall poor state of preservation and incompleteness of the skeletal remains recovered required a detailed macroscopic examination to collect sufficient data to answer the main question posed in this research: whether the social status of the individuals buried at El-Zuma was reflected in their physical health. The observations that were made, essential to run statistical analyses (which were considered useful despite the small sample), contributed to a more in-depth interpretation of the social characteristics of the group that used El-Zuma as a burial ground for their dead.

The methodology applied in this study and the dataset are presented below. The catalogue presents demographic characteristics (sex and age at death), stature, and pathological changes determined in the course of the macroscopic examination. The subsequent analysis was aimed at determining characteristics associated with the three types of graves distinguished at the cemetery (Mahmoud El-Tayeb 2007; Obłuski 2005; for a discussion of tomb types, see Chapter 4, this volume).

\section{$2 \quad$ Material}

The human skeletal remains considered in the present chapter came from 21 tumuli excavated by the field team at El-Zuma between 2007 and 2016. Over the course of three study seasons, in 2013, 2014 and 2016, the author examined bones from burials in 12 tumuli: T.7, T.8, T.9, T.14, T.15, T.16, T.20, T.21, T.22, T.24, T.26, and T.28, excavated between 2011 and 2015. He also took into account the osteological assemblage recovered from nine tumuli (T.5, T.10, T.11, T.13, T.17, T.18, T.19, T.25, and T.27) in 2007 and 2009, and originally studied and published by Aleksandra Pudło (2010).

Of the remaining eight tombs (of a total of 29 tumuli identified at El-Zuma; see Fig. 2.9B, this volume), one (T.29) was not excavated and another (T.12) — the only one without a superstructure — did not yield any remains. ${ }^{1}$ The burial chamber of T.6 is expected to yield human remains once its excavation becomes feasible (Mahmoud El-Tayeb, Skowrońska, and Czyżewska 2016, 123). No human bones were recovered from T.2 (Mahmoud El-Tayeb 2005, 391394), whereas T.23 yielded only a single human bone (Mahmoud El-Tayeb 2005, 394). The nine osseous assemblages previously examined by Aleksandra Pudło (2010) were reexamined by the author in an effort to reduce interobserver error and ensure methodological consistency.

Due to the poor preservation of the bones, the initial examination was conducted in situ, followed by a comprehensive analysis in a field laboratory. All of the excavated tombs had been previously looted, most probably in the distant past. Only some of the bones were found intact, with the bulk of the assemblage being heavily fragmented and badly eroded. The severe mechanical fragmentation resulting from repeated plundering of the graves accelerated the deterioration of the osseous material (Mahmoud El-Tayeb, Skowrońska, and Czyżewska 2016, 110; Mahmoud El-Tayeb 2005, 394). In addition, the exposure of the bones to atmospheric factors and animal scavenging in the open robbers' pits further contributed to the bone degradation, therefore heavily impacting data acquisition. Some bones and bone fragments were found outside the burial chambers, in the tunnels or shafts, likely carried away by scavenging animals or removed with other looted material. Furthermore, the environment in the subterranean burial chambers was humid despite the location of the burial ground on the high northern bank of the Nile. Groundwater from the Nile is not an issue at this height, but rather the surface rain water and the now encroaching human settlement; evidence of the latter is unquestionable at the site today. Another destructive factor besides humidity and plundering is the friability of the highly heterogeneous rock into which the grave substructures were cut. Collapsing ceilings in the main grave chamber have repeatedly caused further serious damage to the skeletal remains.

1 There is a possibility, however slight, that some human remains may yet be found in the unexplored part of the western chamber, although the excavator believes this to be unlikely. 


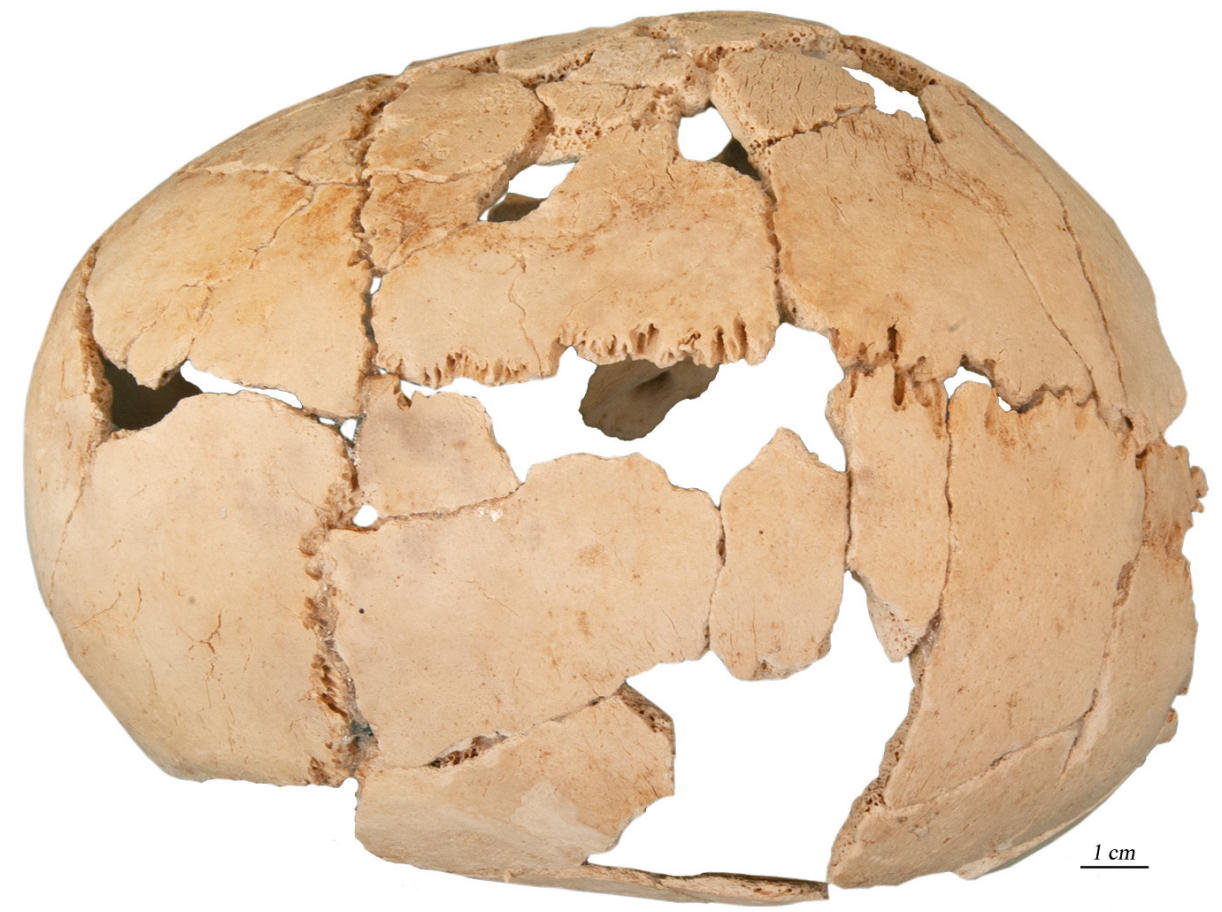

FIGURE 6.1

Reconstruction of the cranial vault of a female (T.14)

PHOTO BY R. MAHLER
From a bioarchaeological perspective, most of the graves from El-Zuma were single burials (a total of 23). The rest contained skeletal remains of multiple burials or the burial content could not be determined. It is not always evident that in the case of single burials the tomb did indeed contain no other inhumations; one cannot be sure that an assemblage of human bones, recovered from a tunnel for example, constituted the original inhumation (or one of several in a multiple burial). It is also likely that the bones could have come from later intrusive burials. The position of the bones was of little help in determining the completeness and number of individuals buried in each tomb. For the most part, the disturbed assemblages could not provide reliable information in this respect, with the exception of the inhumation in T.22, which was sufficiently preserved to establish that the body had been laid "on its right side in contracted position with the head to the south" (Mahmoud El-Tayeb 2005, 398).

\section{3}

\section{Methods}

The workflow of the osteological analysis of bone assemblages from El-Zuma comprised the following:

- Cleaning and reconstruction of the bones in the field laboratory

- Macroscopic analysis and data collection

- Data interpretation from a socio-bioarchaeological perspective.

Statistical methods were used to interpret the data and these require a brief discussion, including the measurement uncertainty involved, to justify the methodology applied and to describe the limitations of the interpretation, as well as the environmental background of the characteristics studied herein.

\subsection{Preparation of the Bones for Analysis}

The poor state of preservation of the skeletons and bone commingling within individual tombs necessitated certain extra measures to prepare the bones for analysis. Since water could not be used for cleaning purposes because of the high degree of bone decay, and because it was hoped to preserve the material for future sampling aimed at laboratory analyses, cleaning the bones involved more laborious, physical procedures.

Observation and measurement of diagnostic elements of the skeleton are essential to collect the data necessary to run certain statistical analyses. To counter the problem of the severe fragmentation of many diagnostic elements, bones were reconstructed wherever possible [Fig. 6.1], using a popular general-purpose, acetone-soluble, thermoplastic adhesive (UHU or a local substitute), with due care not to contaminate clean bone surfaces. This adhesive hardens on the adjoining surfaces without penetrating the bone, even if it is spongy. It is therefore easy to remove and should reduce potential contamination of samples.

The data collected from reconstructed bones are hindered by the same uncertainty that should be taken into account when analysing intact bones in the case of potential post-depositional deformation. 


\subsection{Osteological Analysis}

The methodology applied attempted a compromise between applicability, labour intensity, and potential value of the collected data, the objective being to acquire the most comprehensive dataset that is possible, taking into account the condition of the osseous material and its provenance. Only macroscopic methods of analysis and data collection were selected. A recording form was designed to facilitate subsequent data input into a computer database. The state of preservation of individual skeletons was recorded graphically, allowing skeletal completeness to be visually assessed (Brickley and McKinley 2004, 7, 57-59). Such bone inventory diagrams (see Catalogue below) replace textual descriptions, facilitating immediate comprehension of the recorded material.

The skeletal remains were examined for the following basic characteristics:

1. Number of individuals, using the Minimum Number of Individuals (MNI) method for every single tumulus (White 2000, 291-292), taking into account morphological differences

2. Sex of individuals, using a set of morphological methods compiled by White (200o, 362-371), Piontek (1996, 127-141) and Buikstra with Ubelaker (1994, 16-21)

3. Age at death of individuals, applying a wide range of commonly accepted macroscopic methods:

a) in the case of adults, the set of data taken simultaneously into account when dealing with ageat-death determination consisted of:

- dental attrition, using tables by Lovejoy $(1985,49-50)$

- degeneration of the pubic symphysis: symphyseal face changes by Todd (1921; 1920), after Piontek (1996, 168-169), and Brooks with Suchey (1990), after Buikstra and Ubelaker (1994, 23-24)

- degeneration of the auricular surface: ilium changes by Meindl, Lovejoy and others (Lovejoy et al. 1985; Meindl and Lovejoy 1989), after White (2000, 355-359), further refined by Buckberry and Chamberlain (2002)

- degree of obliteration of cranial sutures, compiled by Buikstra and Ubelaker (1994, 32-35)

b) for juveniles and young adults, the age-atdeath determination was based mainly on the degree of epiphyseal fusion (Piontek 1996, 148) and, when possible, on tooth eruption stages according to tables prepared by Ubelaker (1978,
47), after Piontek (1996, 144-145), along with tables by Al Qahtani, Hector, and Liversidge (2010).

Data collection also included the following, if feasible:

1. Reconstruction of body morphology (Malinowski and Bożiłow 1997; Brothwell 1981; Strzałko 1971; Pearson 1899): height, shoulders-to-pelvic-width proportion, etc. based on long bone measurements (length, circumference, thickness of shafts, width of epiphyses, as well as diameter and circumference of heads). Measurement collection procedures followed generally accepted standards (Buikstra and Ubelaker 1994; Martin and Saller 1959) ${ }^{2}$

2. Evaluation of cranial morphology: measurements of chords (Malinowski and Bożiłow 1997; Buikstra and Ubelaker 1994) and main descriptive features (Piasecki 1992)

3. Evaluation of population homogeneity: so-called epigenetic traits (Alt 1997; Buikstra and Ubelaker 1994; Piontek 1996); most of them should be interpreted as possible reactions to long-lasting external factors, and may be connected, for example, with occupational stress or intentional deformation ${ }^{3}$

4. Evaluation of health and diet based on the following:

a) degenerative changes of joints (Burt et al. 2013; Rogers and Waldron 1995; Buikstra and Ubelaker 1994)

b) dental pathology: caries, enamel hypoplasia, calculus, and attrition (Alt, Rösing, and Teschler-Nicola 1998; Caselitz 1998, Table 3; Hillson 1996; Smith 1984)

c) porotic hyperostosis and cribra orbitalia (Steckel et al. 20o6, 13-14; Buikstra and Ubelaker 1994)

d) other pathological changes, described in nonleading terms (Roberts and Manchester 2005) to ensure verifiable diagnoses (Aufderheide and Rodriguez-Martin 1998; Ortner 2003; Waldron 2009).

\subsection{Socio-bioarchaeological Interpretation}

To address the issue of whether the studied skeletons exhibited different biological characteristics depending on the type of grave in which they were buried, the following key characteristics of the individuals were taken into

2 For a brief list of measurements with references to the most popular handbooks, see Brickley and McKinley (2004, 30); however, note the erroneous labeling of tibia length measurements in this work.

3 See, for example, the formation of external auditory exostoses in divers (Smith-Guzmán and Cooke 2018) or the possible correlation between the intentional deformation of the skull and the formation of intersuturary ossicles (O'Loughlin 2004). 
consideration in the analysis: sex, age at death, and nutritional status (as demonstrated by stature and the presence of cribra orbitalia).

\subsubsection{Stature}

The most useful methods of estimating the stature of archaeological populations from a burial context are those based on the length of the long bones (Mahler 2012, 54). The application of the so-called regression formulae can produce reasonably accurate results. However, selecting the most appropriate set of formulae can prove counterintuitive (Mahler 2018, 198-222; Giannecchini and MoggiCecchi 2008; Kozak 1996). The method of choice would be one developed for a population with limb-bones-tostature proportions as similar as possible to the population under study (Strzałko 1971). It would give the most consistent results in terms of the similarity of estimations when applied to different bone measurements of the same skeleton.

In practice, the El-Zuma material did not lend itself to making such a formal selection of the best regressionbased method. The collected material was not sufficiently complete to apply the kind of statistical analyses known from other studies (e.g. from Europe: Giannecchini and Moggi-Cecchi 20o8, 287; Kozak 1996, 84). Nor was the bone assemblage sufficient for an approach such as the one used in the study of early Islamic Alexandria (Mahler 2018, 204205). The equations published by Trotter and Gleser (1977; 1952) based on Afro-American datasets were chosen for this study. This method performed best for women and only slightly worse for men in the case of early Islamic Alexandria (Mahler 2018, 215-216), ${ }^{4}$ and it was second only to Pearson's (Pearson 1899) in the study from Italy (Giannecchini and Moggi-Cecchi 2008, 288). Regrettably, there are no comparisons of different regression-based staturereconstruction methods used on skeletal series closer in time and place to the El-Zuma burials.

The one exception was a tibia from burial T.10, for which Pearson's formula was used instead of the Trotter and Gleser equation. Use of the Trotter-Gleser stature reconstruction formulae for the tibia should be avoided (White 2000, 372) because the length measurements of this bone by these authors has been shown to most probably be wrong (Jantz, Hunt and Meadows 1995; 1994).

4 For the population of Islamic Alexandria buried in the cemeteries on Kom el-Dikka, the author compared formulae by Christopher B. Ruff et al. (2012, 6o6), Michelle H. Raxter et al. (2008, 150), Mildred Trotter and Goldine C. Gleser (1977, 355; 1958, 120; 1952, 495), and Karl Pearson (1899, 196-197).

\subsubsection{Age at Death}

Age at death is another key element of bioarchaeological analysis. No macroscopic analysis alone can determine the exact chronological age of an individual, but it is feasible to arrive at a biological equivalent in the form of a range. Once this is done as a first step, these ranges established for specific individuals need to be recalculated into uniform categories for counting and comparison. The specificity of osteological methods makes it more appropriate when studying skeletal populations to use uneven age categories, which are better at reflecting the ontogenetic rhythm of human life, rather than the arbitrary regular intervals (e.g. 5 years) used by demographers. For the ElZuma material, six age-at-death categories were applied: $\mathrm{O}-7,7-14,14-2 \mathrm{O}, 2 \mathrm{O}-35,35^{-55}, 55^{-\mathrm{X}} .^{5}$ The overlapping borders emphasize the blurred nature of age determinations in osteology.

The age interval constituting the age-at-death determination of an individual frequently encompassed values from more than one age category. Therefore, the share of a single skeleton with a given age at death in the count of individuals of a particular age category was frequently expressed in real numbers. This share may be defined as the probability of the real age at death (biological not chronological) of an individual from a particular age category [Formula 1]. Therefore, the sum of probabilities of all age categories for each skeleton separately amounted to 1 exactly [Formula 2 ]. ${ }^{6}$

$$
\begin{array}{r}
P_{i}=\frac{\frac{x p_{i}}{e p_{i}-s p_{i}}}{\sum_{j=1}^{n} \frac{x p_{j}}{e p_{j}-s p_{j}}} \\
\sum_{i=1}^{n} P_{i}=1
\end{array}
$$

where:

$P_{i} \quad$ probability of the real age of an individual being within a particular age range, assuming the age-atdeath determination is valid;

$i, j \quad$ subsequent age category in a set of age ranges covering a whole human life;

5 The division adopted here after Malinowski and Bożiłow (1997, 303 ) is very similar to the one proposed by Buikstra and Ubelaker (1994, 9).

6 Therefore, an individual with an age-at-death determination of $30-$ 45 would be counted as 0.40 in the $20-35$ age group and 0.60 in the 35-55 age group (in this case the sum in the denominator for the determination of $P$ equals 0.83 ). 
$s p \quad$ starting point of an age range (in years);

$e p \quad$ end point of an age range (in years);

$x p$ count of years in an age range for a given individual.

\subsubsection{Pathological Conditions}

The bones from the El-Zuma burials bore evidence of numerous pathological conditions (see Catalogue), but owing to the overall poor state of preservation, only two kinds could be recorded systematically enough to be analysed. These are: degenerative joint disease and cribra orbitalia.

Degenerative changes of the joints, that is, osteoarthritis and intervertebral disc disease, were the most commonly observed. However, as the appearance of degenerative changes is age-related in general, the interpretation of their frequencies and severity in the light of the different age profiles observed for the three types of graves would not introduce anything new to the discussion.

The second systematically recorded type of pathological change and the most promising for the analysis at hand was cribra orbitalia, the porosity of the orbital roof, which, if coupled with data on stature, has the potential to add to the information pool on the status differences between the groups under study. This pathological lesion is one of the most frequently recorded in skeletal assemblages worldwide (Walker et al. 2009, 109) and is widely used as an unspecific indicator of physiological stress in the early stages of human life (Lorkiewicz 2012, 131), hence it is very useful in population studies. The lesion was scored on a four-degree scale, from o to 3 (Steckel et al. 2006, 13).

\subsection{Uncertainty of Measurements and Determinations} The error inherent in the study of the El-Zuma material was potentially high and in excess of the uncertainty resulting from the methods employed due to the high degree of fragmentation of the skeletal material and its poor state of preservation. The careful reconstruction of bones mitigated this flaw but did not eliminate it entirely. In the case of long bones, a partial solution to the problem was to approximate measurements wherever feasible and to grade the scale of uncertainty of the results from o to 3. Critical values of uncertainty of the length measurements were arrived at through comparison with complete, undamaged bones. Two groups of maximum absolute uncertainty of estimations were established. The femoral, tibial, fibular, and humeral lengths were found to be burdened by an additional error of $0.0 \mathrm{~cm}, 0.2 \mathrm{~cm}, 0.5 \mathrm{~cm}$, and $1.5 \mathrm{~cm}$ for the successive levels of uncertainty from o to 3 , respectively. Similarly, estimations of the ulnar and radial lengths were found to be burdened by an additional error of $0.0 \mathrm{~cm}, 0.2 \mathrm{~cm}, 0.4 \mathrm{~cm}$, and $1.0 \mathrm{~cm}$ for the respective levels of uncertainty from $\mathrm{O}$ to 3 .

Since the credibility of other determinations, such as sex or degree of cribra orbitalia, was also affected by the generally poor state of preservation of the bones, a threegrade scale (from o to 2) was also implemented to evaluate the levels of uncertainty. However, the same scale carried different meanings for every such feature observed. As it was never transformed into numbers, it should be borne in mind that all it does is to give an indication of the level of uncertainty. Expressed in words, the scale should be read as follows: o-no uncertainty (in excess of the error related to the method applied), 1-low to moderate uncertainty, and 2-high uncertainty, calling for the utmost reserve.

In the case of age at death, the uncertainty of its estimation was reflected in the age range instead.

\subsection{Statistics}

The $\chi^{2}$ test was used to compare proportions wherever essential, following a procedure described, for example, by Drennan (2009, 181-185). Yates's correction for continuity was applied only in cases of at least one cell in a table having an expected count of less than 5 (Zar 2010, 469-470). The calculation was performed using the prop.test $($ ) function of the $\mathrm{R}$ language, particularly useful when comparing proportions with given expected values.

Moreover, the significance of the differences between pairs of means of stature was tested using the two-sided Welch's t-test for two unpaired variables for samples having unequal variances. It was calculated using the t.test() function of the $\mathrm{R}$ language.

\section{$4 \quad$ Results and Discussion}

The minimum number of individuals (MNI) buried in the graves taken into account in this study is $21 .{ }^{7}$ The following table presents their division by tomb type, indicating the sex, age, and stature of the individuals [Table 6.1].

\subsection{Sex}

In the group under study, sex could be determined for 20 individuals. Eight $(38.1 \%)$ females and $12(57.1 \%)$ males were identified in the assemblage. In one case $(4.8 \%)$, the

7 Tumulus 25 was suspected of containing two burials. However, Pudło (2010, Table 1), who studied the bones at the time of discovery, noted only one individual, hence the foot bones that were found by the present author, mixed in with the skeleton from this burial, must have been a later intrusion, possibly already in storage. 
TABLE 6.1 Tumuli annotated by type, with sex, age, and stature of the buried individuals and cribra orbitalia taken into account

\begin{tabular}{llcllll}
\hline & Tumulus & Type (I-III) & Sex & Age $^{\dagger}$ & Stature & Cribra orbitalia $^{\ddagger}$ \\
\hline 1 & T.5 & I & M (?) & $30-50$ & - & 0 \\
2 & T.7 & I & M (?) & $25^{-45}$ & - & 0 \\
3 & T.8 & I & F (?) & $35^{-45}$ & 162 & 2 \\
4 & T.9 & II & M (??) & $25^{-3}$ & 172 & 1 \\
5 & T.11 & II & $?$ & $16-18$ & - & 0 \\
6 & T.13 & II & M (?) & $30-40$ & 175 & 0 \\
7 & T.14 & II & F (?) & $20-30$ & 168 & 2 \\
8 & T.15 & II & F (?) & $21-24$ & 166 & 1 \\
9 & T.16 & II & M (??) & $16-24$ & 167 & 1 \\
10 & T.24 & II & F (?) & $15^{-18}$ & 165 & 2 \\
11 & T.25 & II & F & $24^{-35}$ & 161 & 1 \\
12 & T.26 & II & F & $45^{-55}$ & 164 & 2 \\
13 & T.10 & III & M & $30-40$ & $166^{*}$ & 1 \\
14 & T.17 & III & M & $40-50$ & 173 & 1 \\
15 & T.18 & III & F (?) & $5^{+}$ & 149 & 2 \\
16 & T.19 & III & M (?) & $35^{-45}$ & 167 & 1 \\
17 & T.20 & III & M (??) & $5^{+}$ & 171 & 2 \\
18 & T.21 & III & M & $35^{-40}$ & 167 & 2 \\
19 & T.22 & III & M & $35^{-45}$ & 165 & 0 \\
20 & T.27 & III & M & $35^{-45}$ & 165 & 1 \\
21 & T.28 & III & F & $40-55$ & 160 & 1 \\
\hline & & & & & & \\
\hline & & & & & & 1 \\
\hline
\end{tabular}

$\dagger$ Age-at-death of individuals is given in the intervals that reflect the inherent resolution of applicable methods of age determination.

$\ddagger \quad$ Due to the overall poor state of preservation of the skeletal remains, the cribra orbitalia scores were burdened with uncertainty (for details see Catalogue).

Graves previously studied by Pudło (2010) are marked in grey. Asterisk (*) denotes stature estimation calculated using Pearson's formulae (1899)

PRODUCED BY R. MAHLER

young age of the individual coupled with the poor state of preservation excluded sex determination. The apparent predominance of males in this assemblage, constituting $60 \%$ of the successful sex determinations, may not be entirely true as three of the male determinations are highly uncertain. Moreover, even if this uncertainty were to be overlooked, the $\chi^{2}$ test of proportions shows this difference to be statistically insignificant $\left(\chi^{2}=0.8 ; p=0.3711\right)$ when compared to the ideal 1:1 sex ratio. ${ }^{8}$

The sex ratio in the context of a grave type does not give a clear picture either. Both male and female individuals were buried in structures of all three types. A certain

8 According to Fisher's equal investment principle (Seger and Stubblefield 2002, 9) and as proved by empirical data (Bagnall and Frier 1994, 95), we should expect the ratio of sexes in human populations to be near equal. preference is discernible for burying females in tombs of type II $\left(\mathrm{F}=5 ; \mathrm{M}=3 ; \chi^{2}=0.125 ; p=0.7237\right)$ and males in tombs of type III $\left(\mathrm{F}=2 ; \mathrm{M}=7 ; \chi^{2}=1.7778 ; p=0.1824\right)$, but statistical results do not support these observations; the test of the sex ratio between these grave types also proved the differences to be insignificant $\left(\chi^{2}=1.4175 ; p=0.2338\right)$. Moreover, the sex ratio for tombs of type I, with a count of 3 individuals $(F=1 ; M=2)$, is purely random and should not be taken into account in any discussion until new data on type I burials become available.

\subsection{Age at Death}

Frequencies of age-at-death categories are summed up in tabular form [Table 6.2] and presented in a diagram [Fig. 6.2]. The age-at-death profile of the group buried in these particular tumuli at El-Zuma seems to be representative of a higher social stratum that considered the 
TABLE 6.2 Count of individuals in age-at-death categories, for each grave type separately

\begin{tabular}{llccccc}
\hline & & $\mathbf{1 4 - 2 0}$ & $\mathbf{2 0 -} \mathbf{3 5}$ & $\mathbf{3 5}-\mathbf{5 5}$ & $\mathbf{5 5}-\mathbf{x}$ & $\Sigma$ \\
\hline Type I & $\mathbf{n}$ & 0 & 0.88 & 2.12 & 0 & 3 \\
& $\mathbf{( \% )}$ & $(0)$ & $(29.3)$ & $(70.7)$ & 0 & $(100)$ \\
Type II & $\mathbf{n}$ & 2.71 & 4.86 & 0.76 & 0.67 & 9 \\
& $\mathbf{( \% )}$ & $(30.16)$ & $(53.97)$ & $(8.47)$ & $(7.41)$ & $(100)$ \\
& & & & & & \\
Type III & $\mathbf{n}$ & 0 & 0.57 & 6.83 & 1.6 & 9 \\
& $\mathbf{( \% )}$ & $(0)$ & $(6.35)$ & $(75.87)$ & $(17.78)$ & $(100)$ \\
& & & & & & \\
$\Sigma$ & $\mathbf{n}$ & 2.71 & 6.31 & 9.71 & 2.27 & 21 \\
& $\mathbf{( \% )}$ & $(12.93)$ & $(30.04)$ & $(46.24)$ & $(10.79)$ & $(100)$ \\
& & & & & & \\
\hline
\end{tabular}

PRODUCED BY R. MAHLER

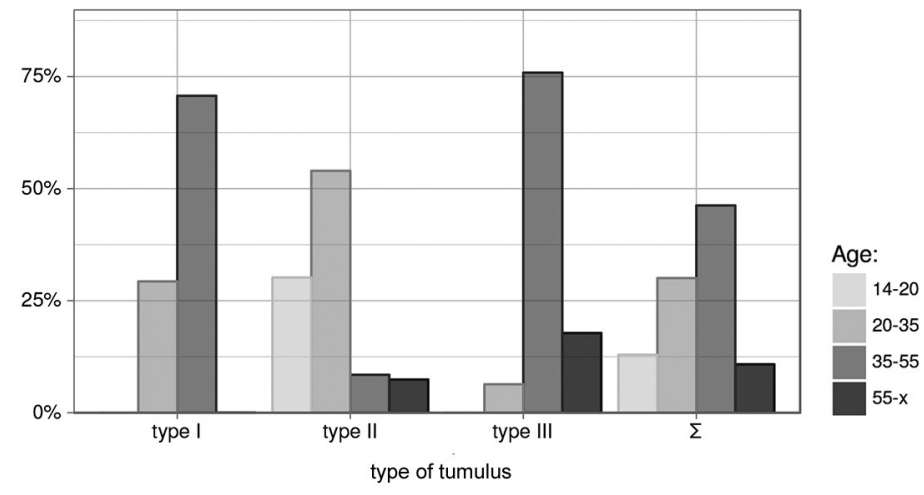

FIGURE 6.2

Frequencies of age-at-death categories of individuals buried in the cemetery taking into account three different tomb types PRODUCED BY R. MAHLER cemetery as their special burial ground (see Chapter 11, this volume).

Looking at the burials without distinguishing them by tomb type, the most numerous group was that of mature individuals ( $35^{-55}$ years of age), followed closely by young adults $(20-35)$. The least numerous were the groups of old adults $\left(55^{+}\right)$and subadults $(14-20)$. None of the individuals buried at this cemetery was younger than 14 years of age (in terms of development age ranges, not actual chronological age).

Tumuli of type III seem to have been constructed for mature individuals only. None of the individuals inhumed in this type of tomb was classified under 30 years of age and some may have even reached an advanced age of $5^{\circ}$ and more. Skeletons recovered from type II tombs represented younger individuals. Most of them $(n=4 ; 4.86$ in terms of frequency, amounting to $53.97 \%$ of all analysed burials of type II) were young adults (20-35 age category), and some of them $(n=3$; with an age-group frequency of 2.71 , that is $30.16 \%$ ) may not have lived to early adulthood. In terms of age at death, the three individuals buried in type I tumuli seem to have been positioned somewhere in between these two extremes of young and old adults.

Moreover, the difference between age-at-death frequencies of type II and type III graves appears to be statistically significant $\left(\chi^{2}=11.335 ; p=0.01005\right)$, but we need to bear in mind the small number of individuals used in this comparison $(n=18)$. Importantly, type II and type III tombs also differ in the composition of sexes and these characteristics appear to be interdependent. One can thus observe a difference between these two types of tombs in terms of the age at death and sex of the individuals inhumed, but the exact nature of this difference cannot be ascertained. ${ }^{9}$

9 As El-Zuma was in all likelihood not a burial ground for a whole community, the observed differences may be cautiously interpreted as reflecting a social hierarchy, in which, for example, type II graves may be assumed to be the tombs of relatives of the most important figures (buried in type I structures), while the type III graves would have been intended for those who earned their higher position rather than being born into it. 
TABLE 6.3 Mean stature of individuals buried at El-Zuma taking into account three grave types and sex

\begin{tabular}{|c|c|c|c|c|c|c|c|c|}
\hline & Sex & $n$ & $x$ & $s$ & $x_{\text {min }}$ & $x_{\max }$ & $\overline{M U E}$ & $\overline{S E E}$ \\
\hline \multirow[t]{2}{*}{ Туре I } & Q & 1 & 162 & - & 162 & 162 & 4.62 & 4.25 \\
\hline & $\sigma^{7}$ & O & - & - & - & - & - & - \\
\hline \multirow[t]{2}{*}{ Type II } & O & 5 & 164.8 & 2.59 & 161 & 168 & 0.68 & 4.12 \\
\hline & $\sigma^{\pi}$ & 3 & 171.3 & 4.04 & 167 & 175 & 1.77 & 4.26 \\
\hline \multirow[t]{2}{*}{ Type III } & Q & 2 & 154.5 & $7 \cdot 78$ & 149 & 160 & 1.71 & 3.41 \\
\hline & $\sigma^{7}$ & 6 & 168 & 3.29 & 165 & 173 & 1.33 & 4.00 \\
\hline
\end{tabular}

$\overline{M U E}$ mean maximum uncertainty of estimation of stature resulting from the uncertainty of the measurements of long bones used in stature estimation

$\overline{S E E}$ mean standard uncertainty of stature estimation resulting from specific regression formulae employed

PRODUCED BY R. MAHLER

\subsection{Stature}

Stature as a characteristic that is relatively very ecosensitive can be used as a good measure of the overall wellbeing of the group under study (Steckel and Rose 2002, 19-22). It works best when compared between groups of similar genetic background, so the potential differences observed can be attributed solely to the differences in extrinsic factors that influenced their growth. As far as the El-Zuma burial ground is concerned, it is very likely that as a group all the deceased were very much alike in terms of genetic characteristics, so the majority of the potential dissimilarities in mean stature may in general be attributed to the differences in the overall well-being of particular subgroups.

Interesting results come from the comparison of means calculated for the stature of individuals buried in the different types of graves identified at El-Zuma. Table 6.3 shows that both males and females buried in the tombs of type II were considerably taller than those from type III burials. Unfortunately, the differences observed were statistically insignificant, most probably due to the small number of available estimations of stature. The t-Student test gave $t=1.2384$ with $p=0.2943$ for males and $t=1.8326$ with $p=0.302$ for females. Even so, the overall well-being of individuals buried in type II structures was most probably higher than that of the type III ones.

For the most monumental tumuli of type I there was only one stature estimation available, that of a female $(162 \mathrm{~cm})$. While the observation is inconsequential because it is singular, it is worth noting that the individual was $2 \mathrm{~cm}$ taller than the maximum $160 \mathrm{~cm}$ obtained for the taller of the two females buried in type III tombs.

\subsection{Cribra Orbitalia}

The role of iron deficiencies, widely accepted as a general factor causing the formation of cribra orbitalia (Lewis 2007, 112-113; Roberts and Manchester 2005, 229-233; Goodman and Martin 2002, 27-29; Larsen 1997, 29-33), has been convincingly questioned in favour of a different diagnosis that indicates haemolytic, congenital, or megaloblastic anaemia, connected with nutritional deficiencies and disturbances in assimilation of vitamin $B_{12}$ as causative factors behind these lesions (Walker et al. 2009). As the discussion continues (Rivera and Mirazón Lahr 2017; Smith-Guzmán 2015, 1; Klaus 2017, 98-101; McIlvaine 2015; Oxenham and Cavill 2010; Wapler, Crubezy, and Schultz 2004), the picture that emerges favours a heterogeneous, multi-factor, both congenital and environmental aetiology of this condition.

The scoring of cribra orbitalia was feasible in 16 cases only. Severe (score 3 ) lesions were not detected and light lesions (score 2 ) were present in seven cases. Of that number, three were found in burials inside type III structures $(n=8)$, three were present in type II $(n=7)$ and one in a type I structure.

There appears to be no observable pattern in the distribution of cribra orbitalia lesions in burials of different types in the El-Zuma tumuli. Therefore, early childhood physiological stress, which causes this condition to be manifested, may have influenced all three groups to the same extent. It is also possible that the limited number of observations, especially in the case of burials from type I tumuli, made it impossible to detect a pattern that in fact existed. 


\subsection{Other Pathological Conditions}

Degenerative changes of the joints, which are the most frequently observed pathological condition affecting human bones, were noted mainly among individuals buried in type III and type I graves. All the individuals from these two groups presented degenerative changes either of the spine or other joints. Since the condition is in general correlated with age (Waldron 2009, 28), it is not surprising that only four out of nine of the generally younger individuals buried in type II graves showed such degenerative lesions.

Changes reflecting oral health are also to some extent age-dependent. Although carious lesions were not observed on teeth examined from the El-Zuma cemetery, some individuals showed periapical changes. Oral health observations were only possible for a total of 15 individuals: one case from a type I grave, six from type II graves, and eight from graves of type III. In the one case from a type I burial, no lesions were found. Among individuals from the type II burials there was one case of a possible abscess and five individuals with no periapical lesions observed. Two of the eight individuals buried in type III graves presented multiple abscesses, the third had only one, and the fourth had two lesions of this kind together with a periapical granuloma. Periapical inflammations may have been caused by excessive caries that resulted in root canal infection. It is also possible that some of the abscesses were caused by other factors, such as periodontal pocket formation (example from T.1o of a deep pocket running down to two-thirds of the root's length) or excessive tooth wear leading to root canal exposure.

There were three possible cases of traumatic lesions detected, but only among individuals buried in type III graves. The individual from T.1o presented a possible healed compression fracture of the body of two thoracic vertebrae. A bony outgrowth on a proximal phalanx of the right foot of the individual in T.22 may attest to a healed injury, while the flat lesion on the frontal bone of the skull in T.27 may have been the result of a blow or impact with a force running parallel to the bone surface (or attempted scraped trepanation interrupted at an early stage of the procedure).

Other lesions were also observed, including porosity of the outer table of the skull (T.8, T.14, T.24, T.21), arachnoid granulations on the inner table of the skull (T.11, T.2O), Schmorl's nodes on the bodies of the lumbar (T.7, T.10) and lower thoracic vertebrae (T.8, T.10), and on the body of the sacrum (T.16), inflammation of the maxillary or frontal sinuses (T.14, T.20, T.22, T.27, T.28), and minor congenital defects, namely a groove (T.11) or a pit (T.26) on the lunate surface of the os coxae, congenital (?) fusion of the left hamate and capitate (T.15), and incisors most probably sharing the same socket (T.17). T.10 had a perforation in the frontal bone, approximately $10 \mathrm{~mm}$ in diameter, with a healed rim (the poor state of preservation prevents a diagnosis).

\section{$5 \quad$ Conclusions}

The results of the osteological study of burials from ElZuma reveal a population with what appears to be a hierarchical social structure. However, the picture drawn here can be supported with statistically significant data only in the case of age-at-death differences.

The hierarchical social structure corresponds well with the three different types of tombs distinguished at the site (Mahmoud El-Tayeb 2007; Obłuski 2005). The building of the most monumental, type I, structures was likely very labour-intensive. Type II tombs were not as grand, but their construction must still have been very expensive. Type II I tombs were simple and required far less labour than the other two types, but their location in the immediate vicinity of the type I and type II structures was most probably an attractive one.

The poorest, type III, tombs were constructed for mature individuals, mostly males, while young individuals, mainly females, were found in most of the type II tombs. Moreover, differences in the proportion of age-atdeath frequencies between the structures of types II and II I were found to be statistically significant. The generally lower social status of those buried in type III structures, assumed because of the least labour required for their construction compared to the other two, was supported with a comparison of mean statures. Nonetheless, the observed differences, although well marked, were statistically insignificant, calling for caution in accepting this conclusion.

The small number of individuals under study and even greater scarcity of data available on their sex, age, stature, and pathological conditions (as observed on bones), imposed research limitations. Certain patterns have been observed linking the biological characteristics of the deceased and the grave structures. Most of them are not reflected in the results of statistical analyses because of the small sample size. The results, however, once they are considered in combination with the outcome of another part of the study, namely isotope analysis conducted by Iwona Kozieradzka-Ogunmakin (Chapter 7 , this volume) and the results of aDNA analysis (see appendix by Abagail Breidenstein at the end of this chapter) should help to shed more light on the people that used the El-Zuma cemetery as the burial ground for their dead. 


\section{Catalogue}

Key:

Asterisk $\left(^{*}\right) \quad$ graves studied and published by Pudło (2010)

MNI Minimum Number of Individuals (White 200o, 291-292)

Sex: $\quad F$ female

M male

? unknown

Diagrams presenting the bone inventory show the elements that were present in grey, those identified but with uncertain siding in blue, and those with uncertain identification and/or siding in black. ${ }^{10}$

Determination uncertainty levels (O-2) are denoted with question marks in brackets following a given value, i.e., no question mark—score o; (?)—score $1 ;(? ?)$ — score 2.

Measurement uncertainty levels (o-3) are denoted with question marks in brackets following a given value (for the methodology, see above), i.e. no question mark-score o; (?) — score 1 ; (??)—score 2 ; and (???)—score 3 .

Permanent teeth were coded according to a generally accepted standard (Alt and Türp 1998, 42, 44-45). Twodigit numbers were used for the teeth: from 11 to 18 and from 21 to 28 for the maxilla, the right and left side respectively, starting from the first incisor, and from 41 to 48 for the right and from 31 to 38 for the left side of the mandible.
Degenerative Joint Disease (DJD):

- OA (Osteoarthritis)_diagnosis follows Waldron's (2009, 34) operational definition

- IDD (Intervertebral Disc Disease)—diagnosis follows Waldron's (2009, 34) operational definition.

Severity of degenerative lesions:

- no mention — no changes or no bone element present (refer to inventory diagram)

- small or light — osteophytes less than about $1 \mathrm{~mm}$, degenerative or productive changes follow Schultz's (1988) score 2, left-hand column after Steckel et al. (2006, 31-33)

- medium or moderate-osteophytes less than about $3 \mathrm{~mm}$, degenerative or productive changes follow Schultz's (1988) score 2, right-hand column after Steckel et al. (2006, 31-33)

- extensive, strong, or severe-osteophytes more than about $3 \mathrm{~mm}$, degenerative or productive changes follow Schultz's (1988) score 3, after Steckel et al. (2006, 31-33).

Cribra orbitalia-scored on a four-level scale (o-3) (Steckel et al. 2006, 13).

For other methodological decisions, see above, section 3.

10 All diagrams are by Marta Momot (Polish Centre of Mediterranean Archaeology, University of Warsaw) based on the author's notes. 
Tumulus 5-type I *

\begin{tabular}{ll}
\hline MNI & 1 \\
Sex & $\mathrm{M}(?)$ \\
Age & $30-50$ \\
Stature & -
\end{tabular}

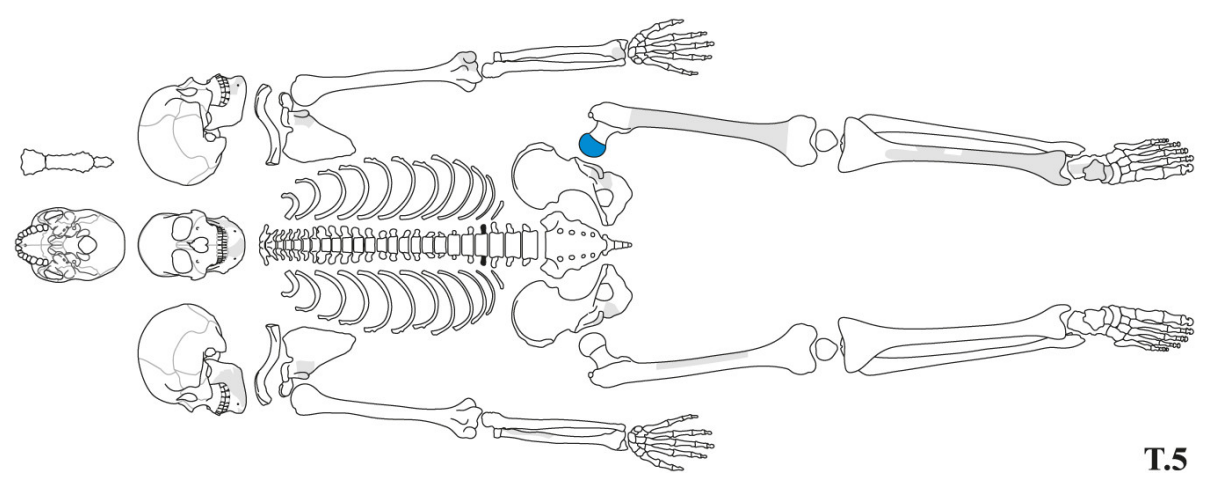

Length measurements (Mi):

bone: humerus radius ulna femur tibia fibula

right: $\quad-\quad-\quad-\quad \begin{array}{lllllll}- & - & - & - & -\end{array}$

left: $\quad-\quad \begin{array}{lllllll} & - & - & - & - & - & -\end{array}$

Dental pathology

None observed.

\section{Skeletal pathology}

DJD: 1) OA: small marginal lipping and small osteophytes on the surface of the distal epiphysis of the left radius cribra orbitalia: 0

other: none observed

\section{Trauma}

None observed.

\section{Other observations}

None. 


\section{Tumulus 7-type I}

\begin{tabular}{ll}
\hline MNI & 1 \\
Sex & $\mathrm{M}(?)$ \\
Age & $25^{-45}$ \\
Stature & - \\
\hline
\end{tabular}

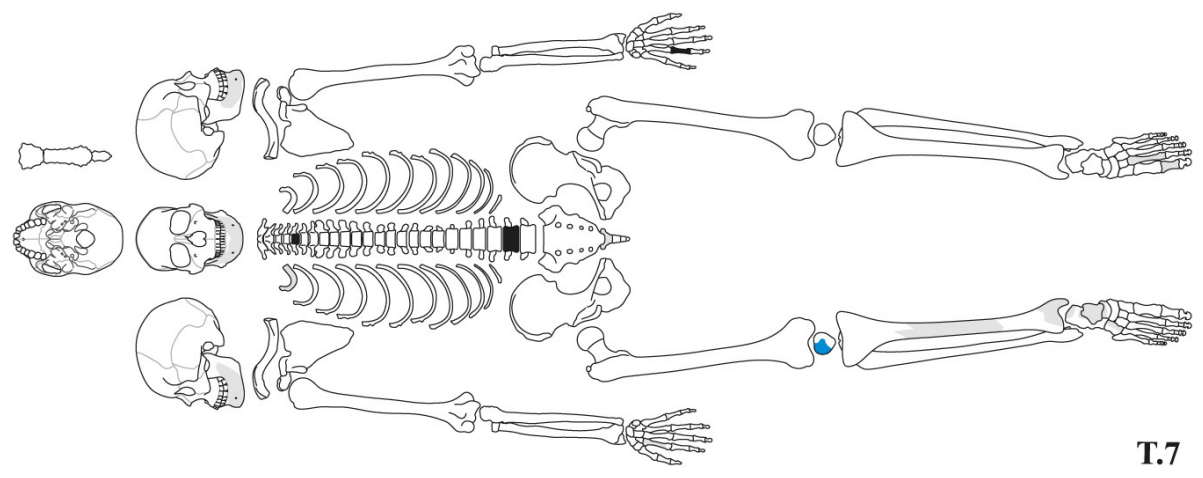

Length measurements (M1):

bone: humerus radius ulna femur tibia fibula

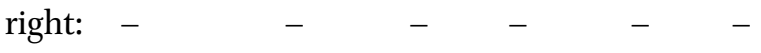

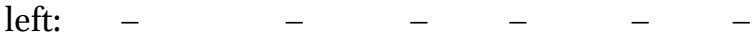

\section{Dental pathology}

None observed.

\section{Skeletal pathology}

DJD: 1) IDD: small marginal osteophytes and small pitting on the superior surface of the body of the lumbar vertebra

cribra orbitalia: 0

other: 1) Schmorl's node on the superior surface of the body of the lumbar vertebra

\section{Trauma}

None observed.

\section{Other observations}

None. 
Tumulus 8-type I

\begin{tabular}{ll}
\hline MNI & 1 \\
Sex & F (?) \\
Age & $35^{-45}$ \\
Stature & 162 \\
\hline
\end{tabular}

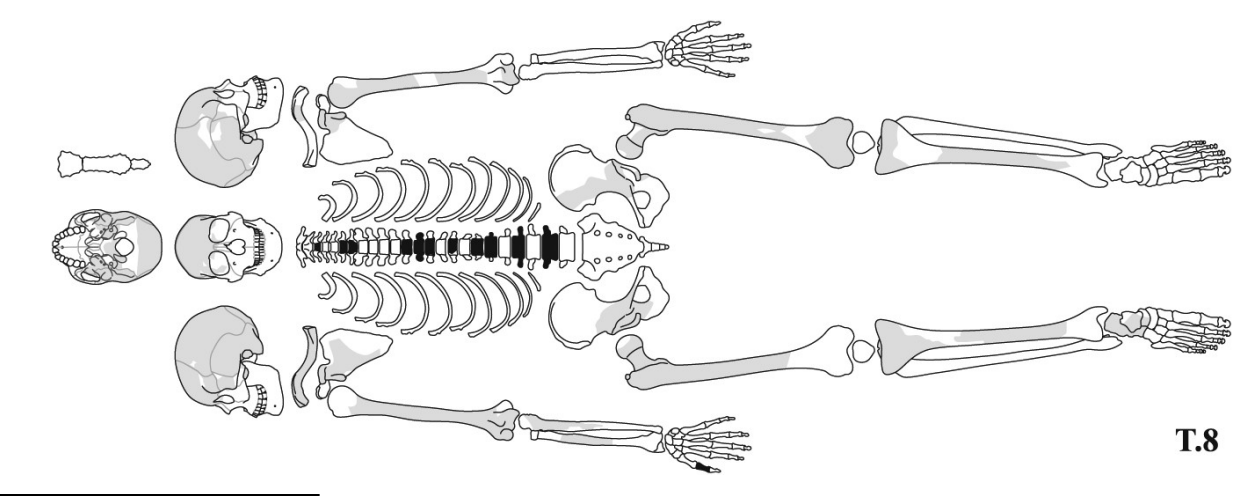

Length measurements (M1):

bone: humerus radius ulna femur tibia fibula

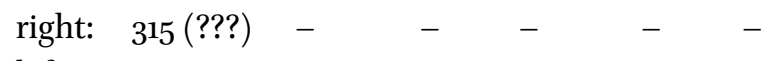



\section{Dental pathology}

None observed.

\section{Skeletal pathology}

DJD: 1) OA: small marginal lipping and small porosity of the lunate surface on the acetabulum of the right os coxae

2) OA: small marginal lipping and moderate pitting of the lunate surface on the acetabulum of the left os coxae

cribra orbitalia: $2(?)$

other: 1) light porosity on the ectocranial surface around the lambda

2) Schmorl's node on the superior body surface of a lower thoracic vertebra

3) small unhealed fracture on the superior surface of a thoracic vertebra

\section{Trauma}

None observed.

\section{Other observations}

None. 
Tumulus 9-type II

\begin{tabular}{ll}
\hline MNI & 1 \\
Sex & M (??) \\
Age & $25^{-30}$ \\
Stature & 172 \\
\hline
\end{tabular}

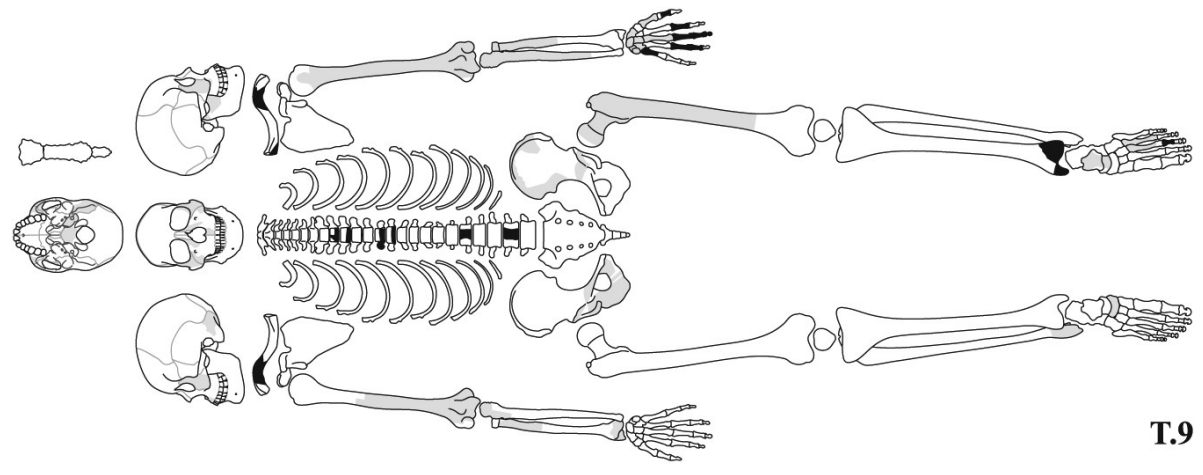

Length measurements (Mi):

bone: humerus radius ulna femur tibia fibula

$\begin{array}{lllllllll}\text { right: } & - & - & & - & - & - & -\end{array}$

left: $\quad 338$ (???) $\quad-\quad \begin{array}{lllllll} & - & - & & - & & -\end{array}$

Dental pathology

None observed.

Skeletal pathology

DJD: none observed

cribra orbitalia: $1(?)$

other: none observed

\section{Trauma}

None observed.

\section{Other observations}

1) 2-mm-wide and 1-mm-deep groove on the lunate surface of the left acetabulum, running next to the rim, parallel to it, for about two-fifths of the length of the lunate surface (probably degenerative lesion) 
Tumulus 11-type II *

\begin{tabular}{ll}
\hline MNI & 1 \\
Sex & $?$ \\
Age & $16-18$ \\
Stature & - \\
\hline
\end{tabular}

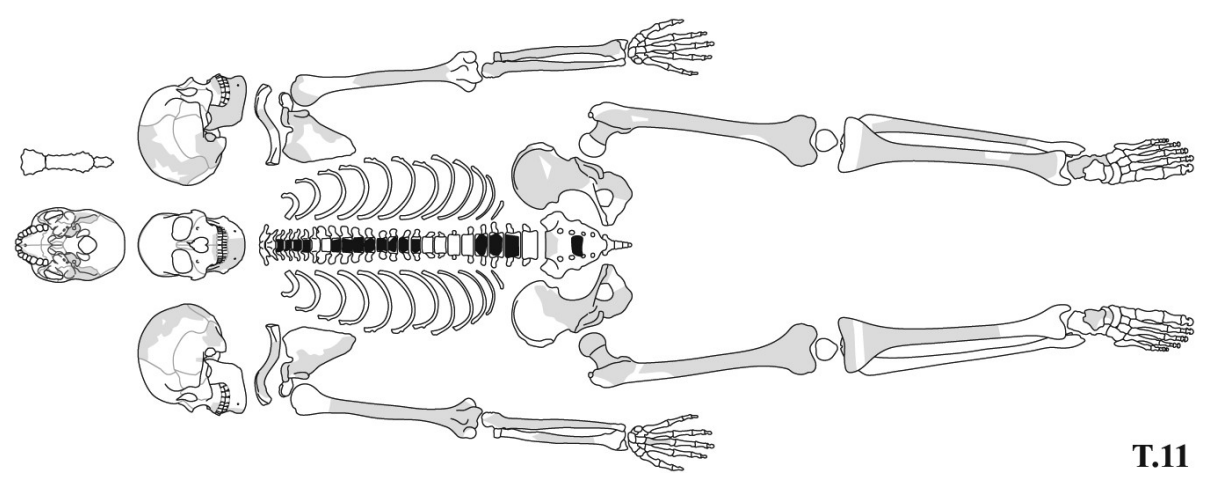

Preserved fragments of:

-8 ribs

Length measurements (Mi):

\begin{tabular}{lllllll}
\hline bone: & humerus & radius & ulna & femur & tibia & fibula \\
right: & - & - & $257(? ? ?)$ & $450(? ?)$ & - & - \\
left: & - & - & - & $455(? ? ?)$ & - & - \\
\hline
\end{tabular}

\section{Dental pathology}

1) a very fine-grained pitting (active) of the bone covering unerupted tooth 48

\section{Skeletal pathology}

DJD: none observed

cribra orbitalia: 0

other: none observed

\section{Trauma}

None observed.

\section{Other observations}

1) congenital dysplasia: groove $1 \mathrm{~mm}$ deep and up to $3.5 \mathrm{~mm}$ wide, bisecting radially the lunate surface of the acetabulum of the left os coxae

2) multiple shallow arachnoid granulations along the sagittal suture, the biggest $19 \mathrm{~mm}$ in diameter 
Tumulus 13-type II *

\begin{tabular}{ll}
\hline MNI & 1 \\
Sex & $\mathrm{M}(?)$ \\
Age & $30-40$ \\
Stature & 175 \\
\hline
\end{tabular}

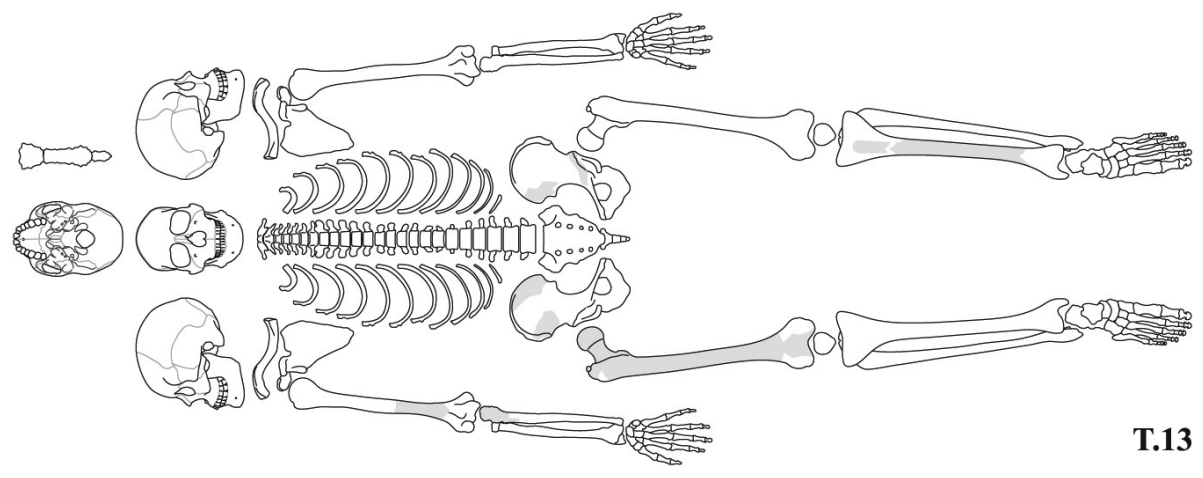

Length measurements $(\mathrm{M} 1)$ :

bone: humerus radius ulna femur tibia fibula

right: - $\quad-\quad-497(?)-4 \quad-$

left: $\quad$ - $\quad$ -

Dental pathology

None observed.

Skeletal pathology

DJD: none observed

cribra orbitalia: 0

other: none observed

\section{Trauma}

None observed.

\section{Other observations}

None. 
Tumulus 14-type II

\begin{tabular}{ll}
\hline MNI & 1 \\
Sex & F (?) \\
Age & $20-30$ \\
Stature & 168 \\
\hline
\end{tabular}

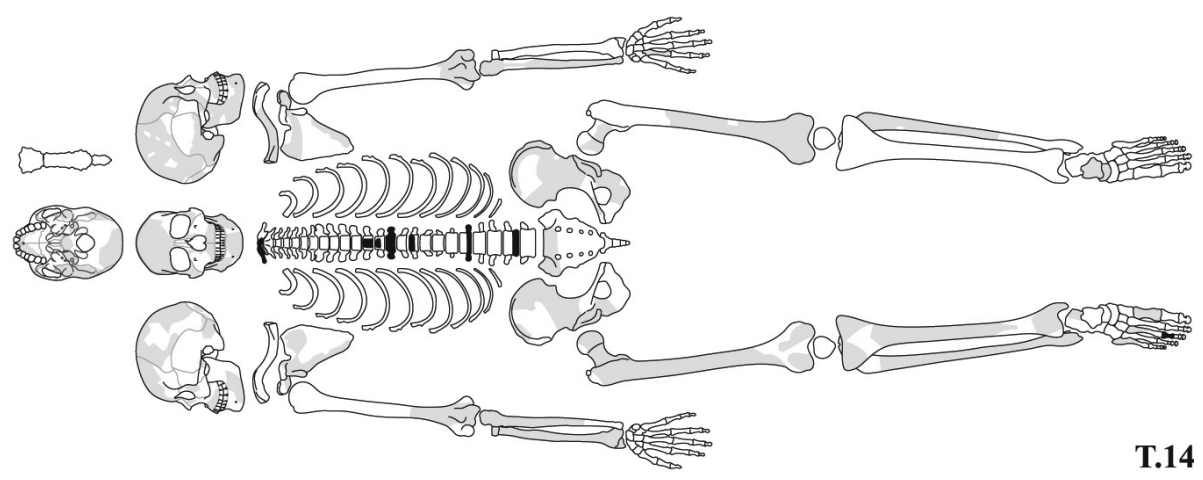

Length measurements (M1):

bone: humerus radius ulna femur tibia fibula

right: - $\quad 262 \quad-\quad 476$ (???) - 394 (???)

left: $\quad-\quad \quad \quad-\quad 284(? ? ?) \quad-\quad \quad \quad-\quad-$

\section{Dental pathology}

None observed.

\section{Skeletal pathology}

DJD: 1) OA: small marginal lipping and small osteophytes in the glenoid cavity of the right scapula cribra orbitalia: $2(?)$

other: 1) light porosity of the outer table of the skull

2) light inactive inflammation of the left frontal sinus

3) cluster ( $7 \mathrm{~mm}$ in diameter) of small osteophytes on the surface of the left glenoid cavity

\section{Trauma}

None observed.

\section{Other observations}

None. 


\section{Tumulus 15-type II}

\begin{tabular}{ll}
\hline MNI & $\mathbf{1}$ \\
Sex & F (?) \\
Age & $21-24$ \\
Stature & 166 \\
\hline
\end{tabular}

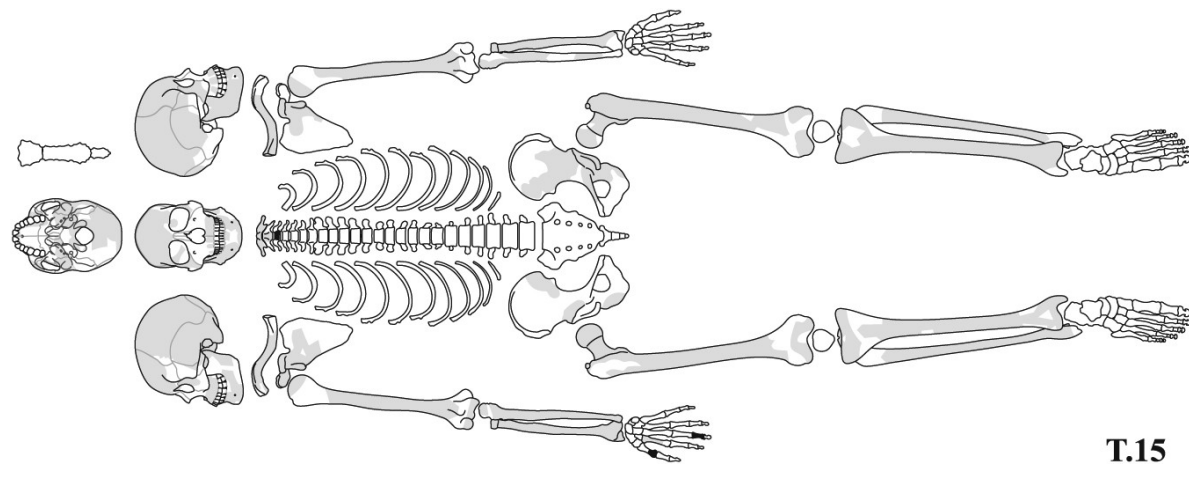

Length measurements (M1):

$\begin{array}{lllllll}\text { bone: } & \text { humerus } & \text { radius } & \text { ulna } & \text { femur } & \text { tibia } & \text { fibula } \\ \text { right: } & - & - & 277(? ? ?) & 463(? ? ?) & - & - \\ \text { left: } & - & 257 & - & - & 387(? ? ?) & - \\ & & & & & \end{array}$

\section{Dental pathology}

None observed.

\section{Skeletal pathology}

DJD: 1) OA: light pitting and small osteophytes on the proximal joint surface of the left tibia and right ulna

2) OA: small marginal lipping and light pitting on the distal joint surface of the left radius cribra orbitalia: $1(?)$

other: 1) congenital (?) fusion of the left hamate and capitate

\section{Trauma}

None observed.

\section{Other observations}

None. 


\section{Tumulus 16-type II}

\begin{tabular}{ll}
\hline MNI & 1 \\
Sex & M (??) \\
Age & $16-24$ \\
Stature & 167 \\
\hline
\end{tabular}

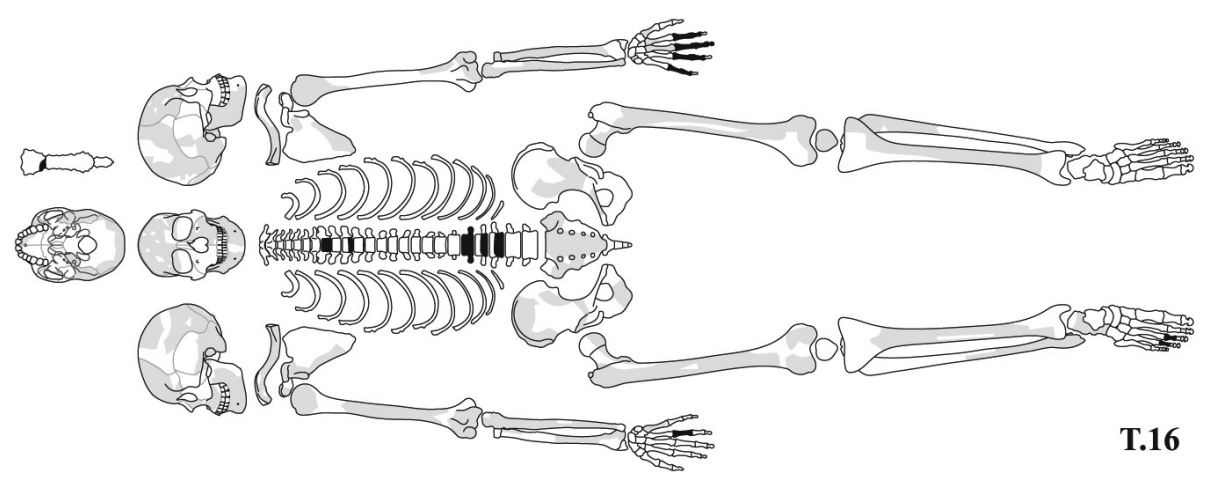

Preserved fragments of:

- 2 foot phalanges

Length measurements (M1):

\begin{tabular}{lllllll}
\hline bone: & humerus & radius & ulna & femur & tibia & fibula \\
right: & - & - & $265(? ? ?)$ & - & $360(? ? ?)$ & - \\
left: & - & $241(? ? ?)$ & 269 & - & $360(? ? ?)$ & - \\
\hline
\end{tabular}

\section{Dental pathology}

1) periapical changes (??) of the socket of tooth 21-alveolar abscess

\section{Skeletal pathology}

DJD: none observed

cribra orbitalia: 1 (?)

other: 1) Schmorl's node on the body of the sacrum

\section{Trauma}

None observed.

\section{Other observations}

None. 


\section{Tumulus 24-type II}

\begin{tabular}{ll}
\hline MNI & 1 \\
Sex & F (?) \\
Age & $15^{-18}$ \\
Stature & 165 \\
\hline
\end{tabular}

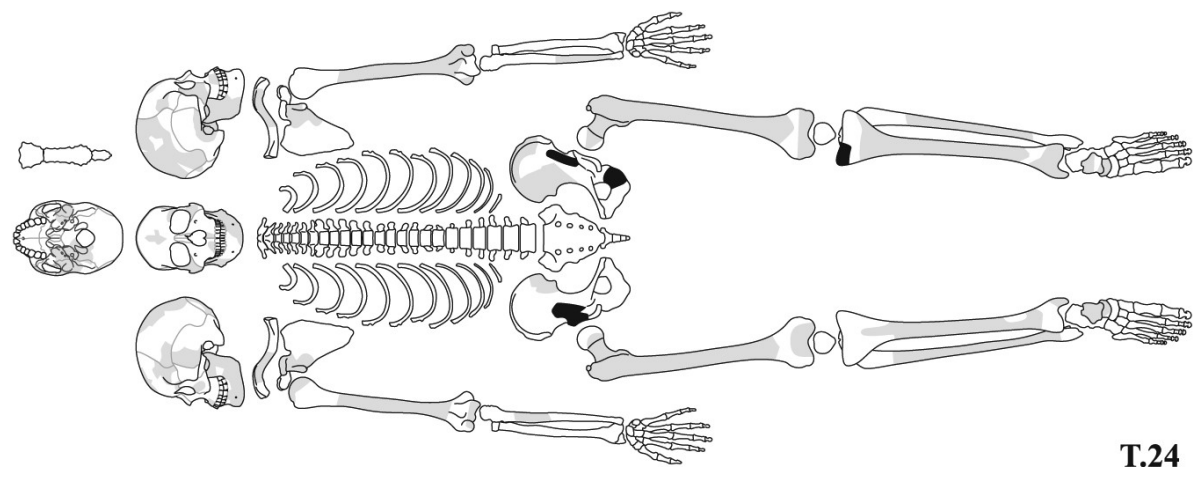

Length measurements $(\mathrm{M} 1)$ :

bone: humerus radius ulna femur tibia fibula

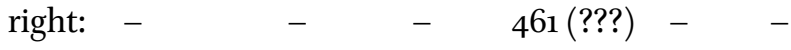

left:

Dental pathology

None observed.

Skeletal pathology

DJD: none observed

cribra orbitalia: 2 (?)

other: 1) light porosity of the outer skull table around the lambda

\section{Trauma}

None observed.

\section{Other observations}

None. 
Tumulus 25-type II *

\begin{tabular}{ll}
\hline MNI & 1 \\
Sex & F \\
Age & $24-35$ \\
Stature & 161 \\
\hline
\end{tabular}



Preserved fragments of:

-13 ribs

- 4 hand phalanges

-6 foot phalanges

Length measurements (Mi):

\begin{tabular}{lllllll}
\hline bone: & humerus & radius & ulna & femur & tibia & fibula \\
right: & $308(? ?)$ & - & - & 444 & - & - \\
left: & - & - & - & $452(? ? ?)$ & - & - \\
\hline
\end{tabular}

\section{Dental pathology}

None observed.

\section{Skeletal pathology}

DJD: 1) OA: small marginal lipping and small pitting on the surface of the left mandibular condyle

2) OA: small marginal lipping and small osteophytes on the surface of the left TMJ articular tubercle

3) OA: small marginal lipping and small porosity of the lunate surface on acetabulum of the left os coxae

4) IDD: small marginal lipping and small pitting of the superior surface of the bodies of the cervical vertebrae $\left(\mathrm{C}_{4}, \mathrm{C}_{5}\right)$

cribra orbitalia: $1(? ?)$

other: none observed

\section{Trauma}

None observed.

\section{Other observations}

1) bones of both feet of a second individual — possible case of post-excavation accidental commingling 
Tumulus 26-type II

\begin{tabular}{ll}
\hline MNI & 1 \\
Sex & F \\
Age & 45 \\
Stature & 164 \\
\hline
\end{tabular}

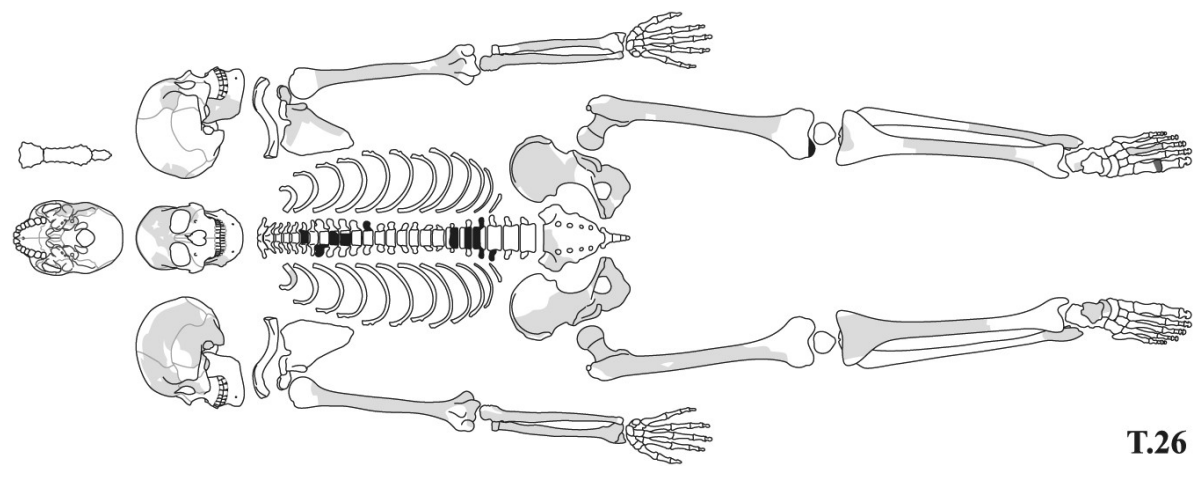

Preserved fragments of:

-3 ribs

Length measurements (M1):

\begin{tabular}{lllllll}
\hline bone: & humerus & radius & ulna & femur & tibia & fibula \\
right: & $324(? ?)$ & 251 & $257(? ? ?)$ & - & - & - \\
left: & - & - & - & - & - & -
\end{tabular}

\section{Dental pathology}

None observed.

\section{Skeletal pathology}

DJD: 1) OA: small marginal lipping, light pitting and small osteophytes on the surface of the right mandibular fossa

2) OA: small marginal lipping, moderate pitting and medium osteophytes on the surface of the right TMJ articular tubercle

3) OA: small marginal lipping and small porosity of the lunate surfaces on the acetabulum of both ossa coxae

4) OA: small marginal lipping and small osteophytes on the distal joint surface of the left fibula

5) OA: light pitting and small osteophytes on the proximal joint surface of the right tibia

6) OA: medium marginal lipping and small osteophytes on the proximal joint surface of the left ulna

7) OA: medium marginal lipping, light pitting and extensive osteophytes on the distal joint surface of a femur (not sided)

8) OA: small marginal lipping, moderate pitting and medium osteophytes on the distal joint surface of the 1st metatarsal (not sided)

9) IDD: small marginal lipping and extensive pitting of the superior body surface of a lower cervical vertebra

10) IDD: medium marginal osteophytes and light pitting on the superior surface of the sacral body

11) OA: small marginal lipping and medium pitting of the right superior articular process, and medium marginal lipping, light pitting and medium osteophytes of the right inferior articular processes of the upper thoracic vertebrae

12) OA: small marginal lipping and small osteophytes of the left inferior articular process of an upper lumbar vertebra

cribra orbitalia: $2(?)$

other: 1) medium osteophytes on the posterior part of the outer rim of the right acetabulum

2) numerous active inflammatory changes of the entheses (enthesitis)

\section{Trauma}

None observed.

\section{Other observations}

1) congenital dysplasia: small pit in the middle of the lunate surface on the acetabulum of the right os coxae [Fig. 6.3]

2) small exostosis on the anterior surface of the distal epiphysis of the right fibula 
3) $3 \cdot 5^{-5}$-mm-wide right emissarium parietale with thinning of the os parietalis in the area-thinning more pronounced on the internal surface [Fig. 6.4]



FIGURE 6.3

Congenital dysplasia; small pit in the centre of the lunate surface on the acetabulum of the right os coxae (T.26) PHOTO BY R. MAHLER

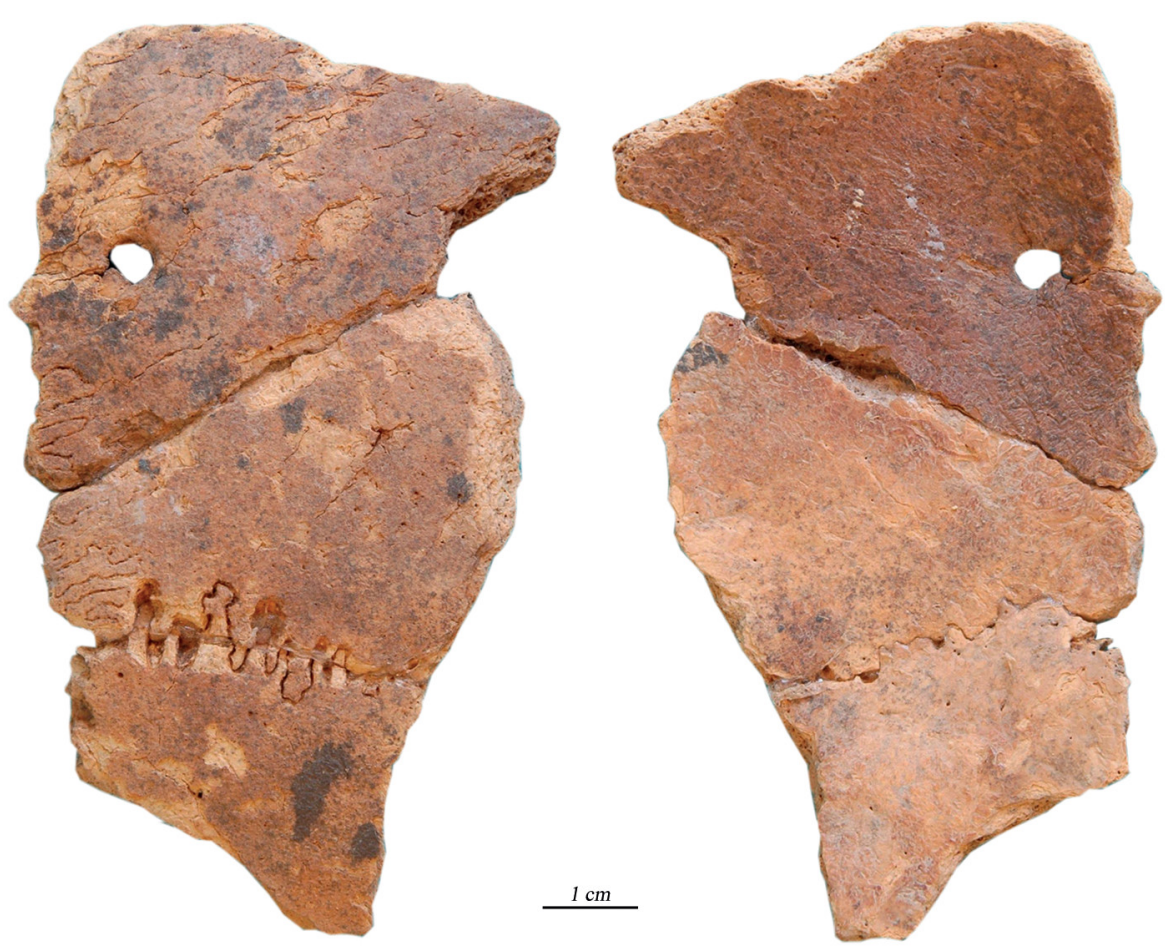

FIGURE 6.4

Emissarium parietale $\left(3 \cdot 5^{-5} \mathrm{~mm}\right.$ wide) with thinning of the right os parietalis in the affected area (T.26) PHOTO BY R. MAHLER 
Tumulus 10-type III *

\begin{tabular}{ll}
\hline MNI & 1 \\
Sex & M \\
Age & $30-40$ \\
Stature & 166 \\
\hline
\end{tabular}

Stature estimation calculated using Pearson's formulae (1899)

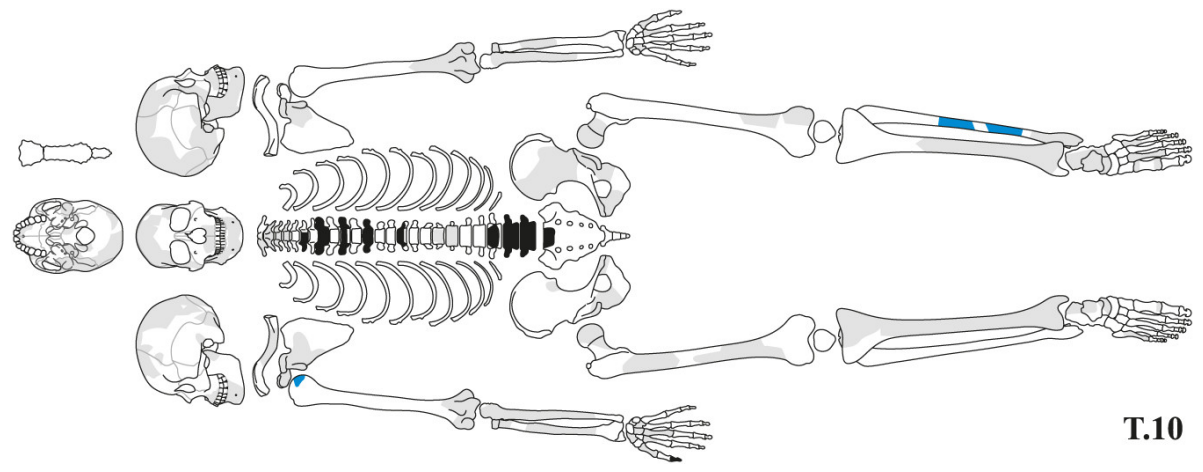

Preserved fragments of:

-3 ribs

- 3 hand phalanges

Length measurements (M1):

\begin{tabular}{lllllll}
\hline bone: & humerus & radius & ulna & femur & tibia & fibula \\
right: & - & - & - & - & $367(?)$ & - \\
left: & - & - & - & - & - & - \\
\hline
\end{tabular}

\section{Dental pathology}

1) periodontal pocket between tooth 47 and tooth 48 , penetrating deep along the root of 48 for two-thirds of its length

\section{Skeletal pathology}

DJD: 1) OA: small marginal lipping and small pitting on the surface of the left mandibular fossa

2) OA: small marginal lipping and medium osteophytes on the distal joint surface of the left fibula

3) OA: small marginal lipping and small porosity of the lunate surface on the acetabulum of the left os coxae

4) OA: medium pitting and small osteophytes of the glenoid fossa of the right scapula

5) OA: small marginal lipping and small pitting of the glenoid fossa of the left scapula

6) IDD: medium-to-large marginal osteophytes and small-to-medium pitting of the superior and inferior surfaces of the body of the lumbar vertebrae

cribra orbitalia: 1 (??)

other: 1) Schmorl's nodes on the lumbar and lower thoracic vertebrae

\section{Trauma}

1) possible case of a compression fracture of the T11 (?) and T12 (?) vertebrae

\section{Other observations}

1) round hole (10 mm in diameter) with healed rim in the upper left part of the frontal skull bone [Fig. 6.5]

2) low bone density of the vertebrae 


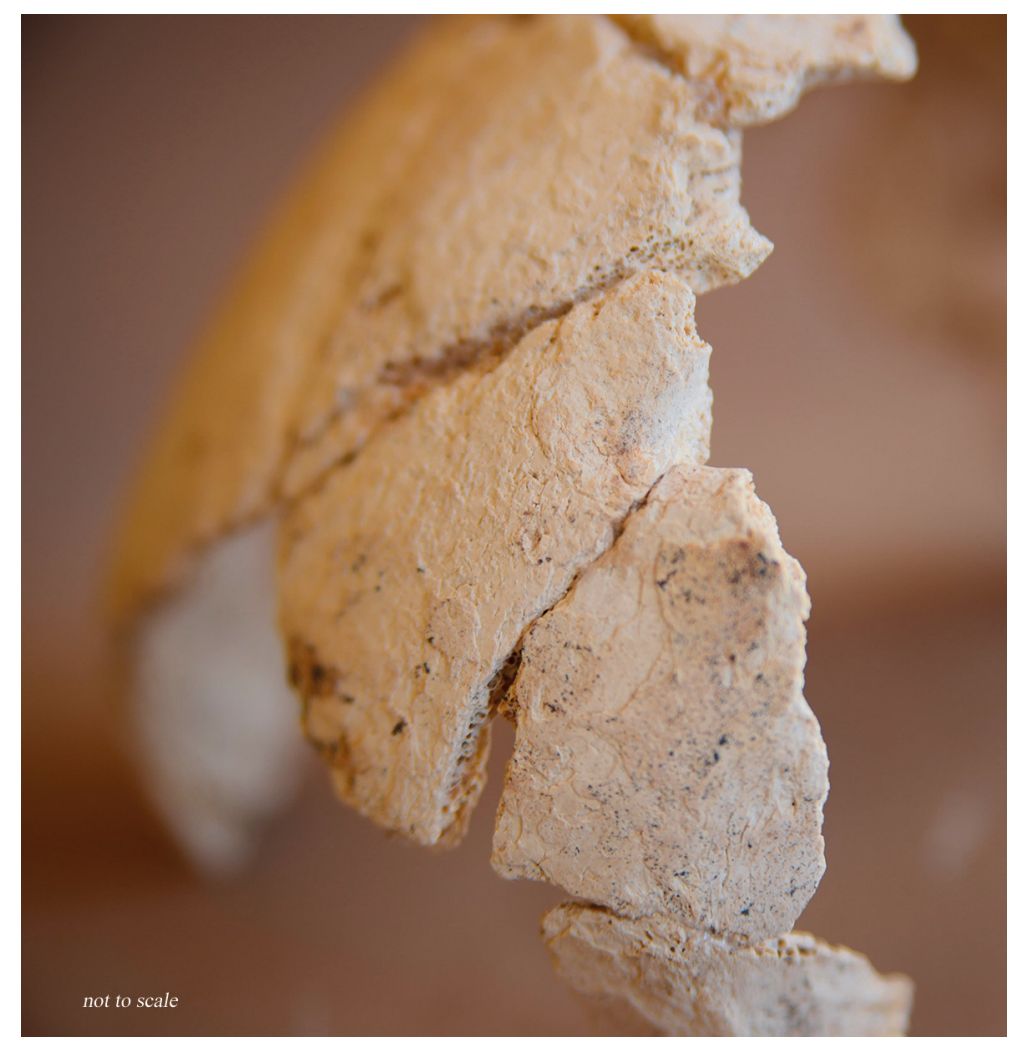

FIGURE 6.5

A round hole (10 $\mathrm{mm}$ in diameter) with healed rim (T.10) PHOTO BY R. MAHLER 
Tumulus 17-type III *

\begin{tabular}{ll}
\hline MNI & 1 \\
Sex & M \\
Age & $40-50$ \\
Stature & 173 \\
\hline
\end{tabular}

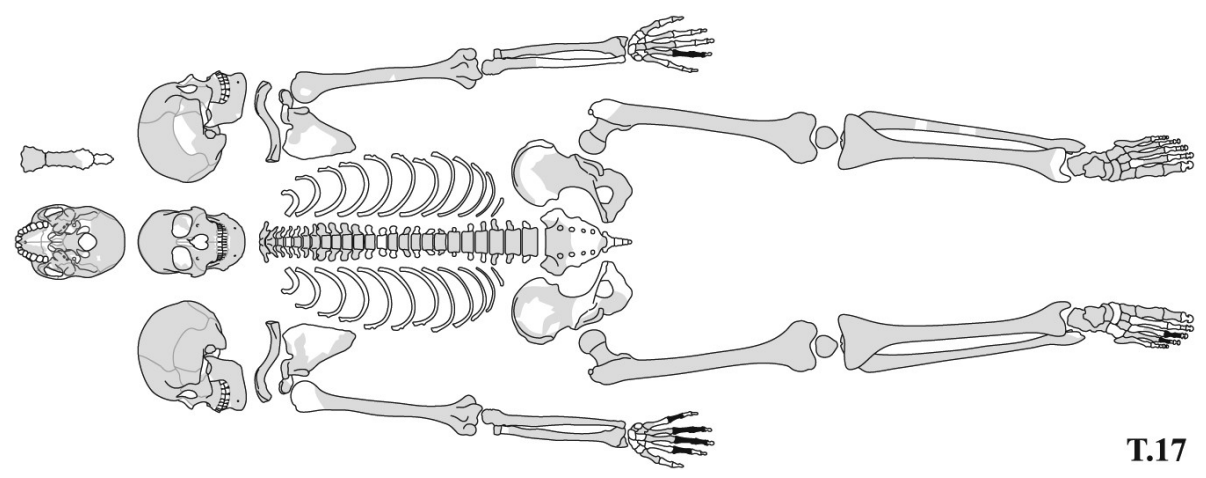

Preserved fragments of:

-15 ribs

- 1 foot phalanx

Length measurements (Mi):

\begin{tabular}{lllllll}
\hline bone: & humerus & radius & ulna & femur & tibia & fibula \\
right: & - & - & 302 & 487 & $420(?)$ & $416(?)$ \\
left: & $349(? ?)$ & 282 & $305(?)$ & 490 & $418(?)$ & - \\
\hline
\end{tabular}

\section{Dental pathology}

1) congenital: teeth 31 and 32 most probably shared the same socket (?) [Fig. 6.6]

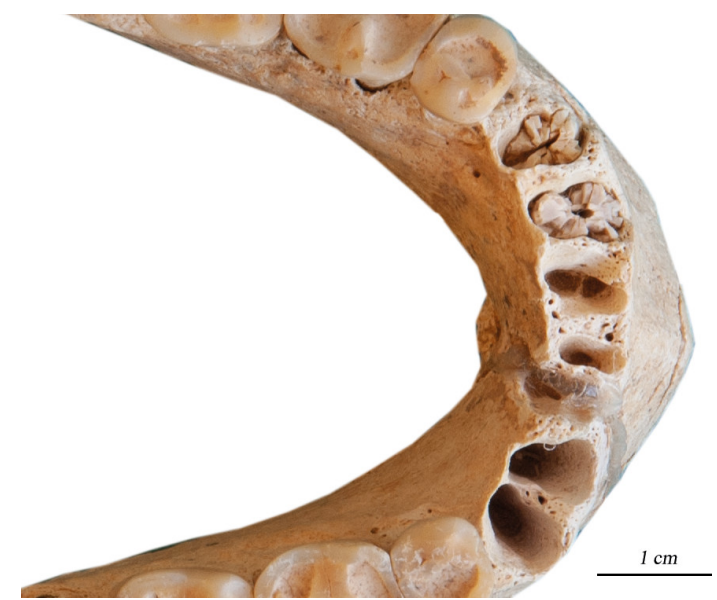

FIGURE 6.6

Teeth 31 and 32 most probably sharing the same socket (T.17) PHOTO BY R. MAHLER

\section{Skeletal pathology}

DJD: 1) OA: small marginal lipping and small osteophytes on the surface of the left TMJ articular tubercle

2) IDD: marginal lipping, small (C6) to moderate $\left(\mathrm{C}_{5}\right)$, and pitting of the superior and inferior surface of the body of the cervical vertebrae, small $\left(\mathrm{C}_{6}\right)$ to severe $\left(\mathrm{C}_{5}\right)$

3) IDD: small marginal lipping and small pitting of the superior and inferior surface of the body of the middle thoracic vertebrae

cribra orbitalia: 1 (?)

other: 1) partial synostosis of both sacroiliac joints (recorded by Pudło, but not observed in the current state of preservation)

\section{Trauma}

None observed.

\section{Other observations}

1) moderate-to-heavy attrition and compensatory eruption of teeth 
Tumulus 18-type III *

\begin{tabular}{ll}
\hline MNI & 1 \\
Sex & F (?) \\
Age & 50 \\
Stature & 149 \\
\hline
\end{tabular}



Length measurements (M1):

\section{Dental pathology}

1) periapical changes of the socket of tooth $24-$ alveolar abscess

\section{Skeletal pathology}

DJD: 1) OA: small marginal lipping and small pitting on the surface of both mandibular fossae

2) OA: light pitting and medium osteophytes on the joint surface of the right patella

3) OA: light pitting and small osteophytes on the surface of the proximal joints of the hand phalanges

4) OA: light pitting and small osteophytes on the right superior and inferior articular facets of the atlas cribra orbitalia: 2 (?)

other: none observed

\section{Trauma}

None observed.

\section{Other observations}

1) bones very light and brittle 
Tumulus 19-type III *

\begin{tabular}{ll}
\hline MNI & 1 \\
Sex & M (?) \\
Age & $35^{-45}$ \\
Stature & 167 \\
\hline
\end{tabular}

Length measurements (M1):

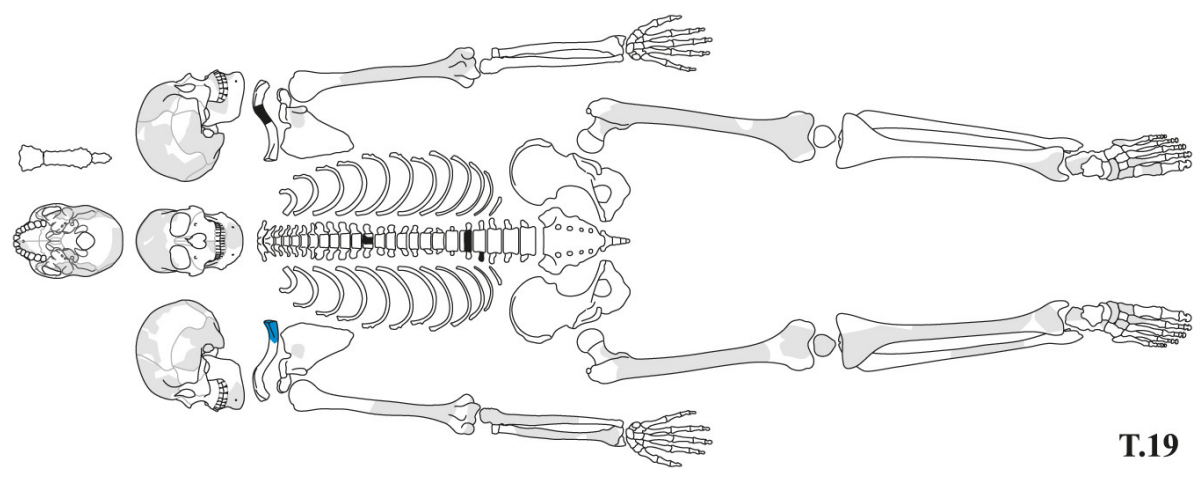

\begin{tabular}{lllllll} 
bone: & humerus & radius & ulna & femur & tibia & fibula \\
right: & - & - & - & - & $390(? ? ?)$ & - \\
left: & - & - & - & $456(? ? ?)$ & - & - \\
\hline
\end{tabular}

\section{Dental pathology}

1) periapical changes of tooth sockets 13,32 (?), 33, 34, 35-alveolar abscess

\section{Skeletal pathology}

DJD: 1) OA: small marginal lipping, moderate pitting and small osteophytes on the surface of the right TMJ articular tubercle

2) OA: small marginal lipping, light pitting and small osteophytes on the distal joint surface of the left femur

3) OA: light pitting and small osteophytes on the joint surfaces of the calcanei

4) IDD: medium marginal osteophytes and moderate pitting of the inferior (?) surface of the body of the lumbar vertebra

cribra orbitalia: 1 (?)

other: none observed

\section{Trauma}

None observed.

\section{Other observations}

None. 
Tumulus 20-type III

\begin{tabular}{ll}
\hline MNI & 1 \\
Sex & M (??) \\
Age & 50 \\
Stature & 171 \\
\hline
\end{tabular}



Preserved fragments of:

- 3 foot phalanges

Length measurements (M1):

\begin{tabular}{lllllll}
\hline bone: & humerus & radius & ulna & femur & tibia & fibula \\
right: & $333(? ?)$ & $268(? ? ?)$ & - & - & - & - \\
left: & $331(? ? ?)$ & - & - & $471(? ? ?)$ & $398(? ? ?)$ & $400(? ? ?)$
\end{tabular}

\section{Dental pathology}

None observed.

\section{Skeletal pathology}

DJD: 1) OA: small marginal lipping and light pitting on the distal joint surface of the left humerus and right radius

2) OA: medium marginal lipping and light pitting on the distal joint surface of the left femur

3) IDD: small marginal lipping and severe pitting of the superior body surface of the lower cervical vertebra and of the inferior body surface of an upper lumbar vertebra

4) OA: small-to-medium marginal lipping and light-to-moderate pitting of the articular processes of thoracic vertebrae (in one case small osteophytes observed as well)

5) OA: small marginal lipping and small osteophytes on a right articular process of an upper lumbar vertebra and on a left articular process of the sacrum

6) OA: medium marginal lipping and light productive changes of interphalangeal joints of both great toes cribra orbitalia: 2 (?)

other: 1) light active inflammation and moderate inactive inflammatory changes of the left maxillary sinus

2) light inactive inflammation of the right maxillary and left and right frontal sinuses

\section{Trauma}

None observed.

\section{Other observations}

1) multiple arachnoid granulations on the inner table of the cranial vault along the sagittal suture, amalgamating into greater depressions in the lambda area, the biggest one $20 \mathrm{~mm}$ by $10 \mathrm{~mm}$

2) moderate-to-heavy attrition and compensatory eruption of teeth 
Tumulus 21-type III

\begin{tabular}{ll}
\hline MNI & $\mathbf{1}$ \\
Sex & M \\
Age & $35^{-40}$ \\
Stature & 167 \\
\hline
\end{tabular}

Preserved fragments of:

-7 ribs



Length measurements (M1):

\begin{tabular}{lllllll}
\hline bone: & humerus & radius & ulna & femur & tibia & fibula \\
right: & - & - & $276(? ? ?)$ & 458 & $389(? ?)$ & 386 \\
left: & - & 262 & - & 455 & $394(?)$ & - \\
\hline
\end{tabular}

\section{Dental pathology}

None observed.

\section{Skeletal pathology}

DJD: 1) OA: strong marginal lipping and light pitting (?) of the head of the left 11th or 12th rib

2) OA: small marginal lipping, light pitting and small osteophytes on the surface of the head of the right femur

3) OA: small marginal lipping and light pitting on the distal joint surface of the left tibia, left radius and right femur

4) OA: medium (right) to small (left) marginal lipping and light pitting of the lunate surface of the acetabulae

5) OA: small marginal lipping and light pitting (10 mm in diameter) on the upper joint surface of the left talus head

cribra orbitalia: $2(?)$

other: 1) light porosity of the ectocranium along the sagittal suture

\section{Trauma}

None observed.

\section{Other observations}

None. 
Tumulus 22-type III

\begin{tabular}{ll}
\hline MNI & 1 \\
Sex & M \\
Age & $35^{-45}$ \\
Stature & 165 \\
\hline
\end{tabular}



Preserved fragments of:

- 10 ribs

Length measurements (M1):

\begin{tabular}{lllllll}
\hline bone: & humerus & radius & ulna & femur & tibia & fibula \\
right: & - & $245(? ? ?)$ & - & - & - & - \\
left: & - & - & - & $450(? ? ?)$ & - & - \\
\hline
\end{tabular}

\section{Dental pathology}

None observed.

\section{Skeletal pathology}

DJD: 1) OA: small marginal osteophytes and light pitting on the surface of the distal joints of the foot phalanges

2) OA: small marginal osteophytes, medium pitting and small osteophytes on the surface of the inferior and superior articular processes of the upper thoracic vertebrae

3) OA: marginal osteophytes and pitting of the articular processes of the lumbar L3 vertebra (small on the superior and medium on the inferior processes)

cribra orbitalia: 0

other: 1) medium-size osteophytes on the anterior surface of the L3 vertebral body

2) light inactive inflammation of the right maxillary sinus

\section{Trauma}

1) bony outgrowth on the dorsal shaft surface of the $\operatorname{3rd}($ ?) proximal phalanx of the right (?) foot—possible trauma [Fig. 6.7]

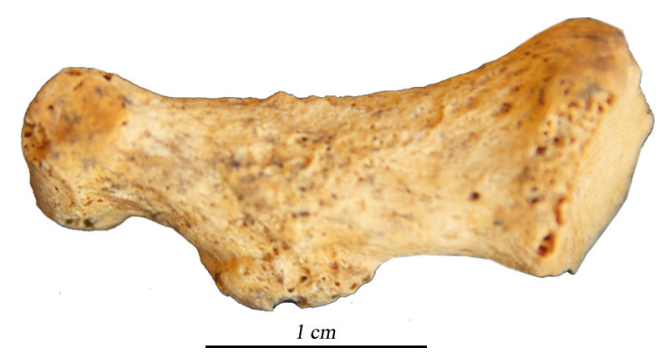

FIGURE 6.7

Proximal phalanx of the right (?) foot (T.22), possible trauma PHOTO BY R. MAHLER

\section{Other observations}

1) moderate-to-heavy attrition and compensatory eruption of teeth (pronounced particularly in the maxilla)

2) symmetrical fovea (pits) on the proximal joint surfaces of both 2nd metacarpals 
Tumulus 27-type III *

\begin{tabular}{ll}
\hline MNI & 1 \\
Sex & M \\
Age & $35-45$ \\
Stature & 165 \\
\hline
\end{tabular}

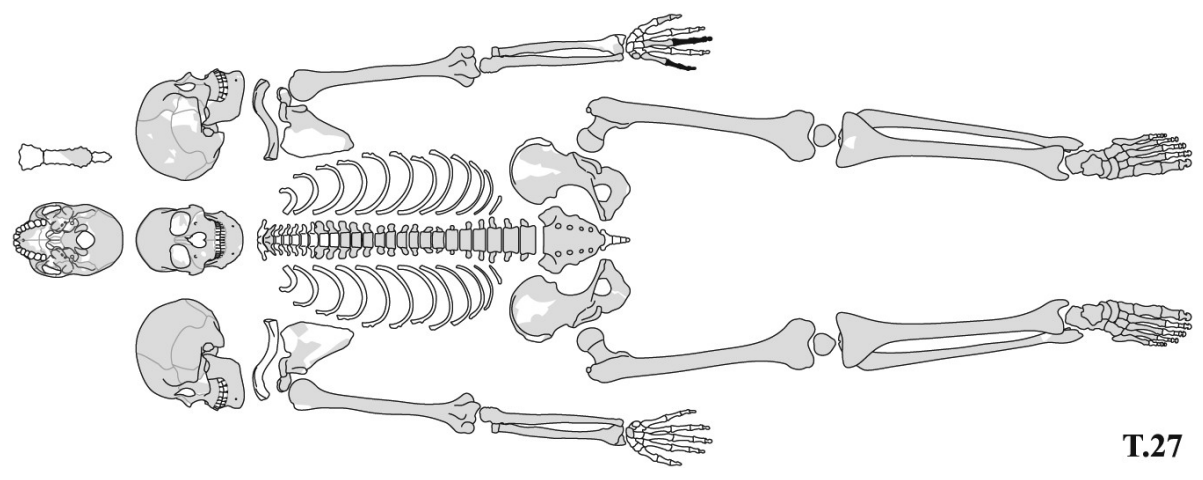

Preserved fragments of:

-12 ribs

Length measurements (M1):

\begin{tabular}{lllllll}
\hline bone: & humerus & radius & ulna & femur & tibia & fibula \\
right: & 324 & $25^{2}$ & 274 & 447 & $378(?)$ & 370 \\
left: & 325 & - & 277 & - & $373(? ?)$ & $367(?)$ \\
\hline
\end{tabular}

\section{Dental pathology}

1) apical granuloma at the position of tooth 23

2) possible abscesses at the position of teeth 37 and 38 (??)

\section{Skeletal pathology}

DJD: 1) OA: medium marginal lipping and light pitting of the lunate surface of the left acetabulum

2) OA: medium marginal lipping, light pitting and small osteophytes in the glenoid cavity of the left scapula

3) OA: small marginal lipping and light pitting on the proximal joint surface of the right tibia and on the distal joint surface of the left tibia

4) OA: light pitting and small osteophytes on the distal joint surface of the left ulna

cribra orbitalia: $1(?)$

other: 1) small button osteoma on the outer table of the left parietal bone, located next to the coronal suture in the middle of its length

2) possible case of a supernumerary left (?) cervical rib (??), most probably fused with the vertebra

3) small exostoses on the right lunate

4) localized lipping (joint surface expansion) on the lower margin of the right femoral head

5) light inactive inflammation of the left frontal sinus

6) irregular contour of the right acetabulum [Fig. 6.8] and light pitting of the lunate surface

7) considerable overgrowth of the lateral process of both calcanei [Fig. 6.9]

\section{Trauma}

1) possible case of healed trauma (?): round irregularity (3-4 $\mathrm{cm}$ in diameter) on the surface of the frontal bone in the centre of the forehead; the impact of the blow (?) appears to have been directed from the upper-right to the lowerleft side of the forehead at an angle of about 25 degrees from the vertical (it may also be interpreted, for example, as attempted scraped trepanation interrupted at an early stage of the procedure)

\section{Other observations}

1) very thin walls of the maxillary sinuses

2) moderate-to-heavy attrition and compensatory eruption of teeth 


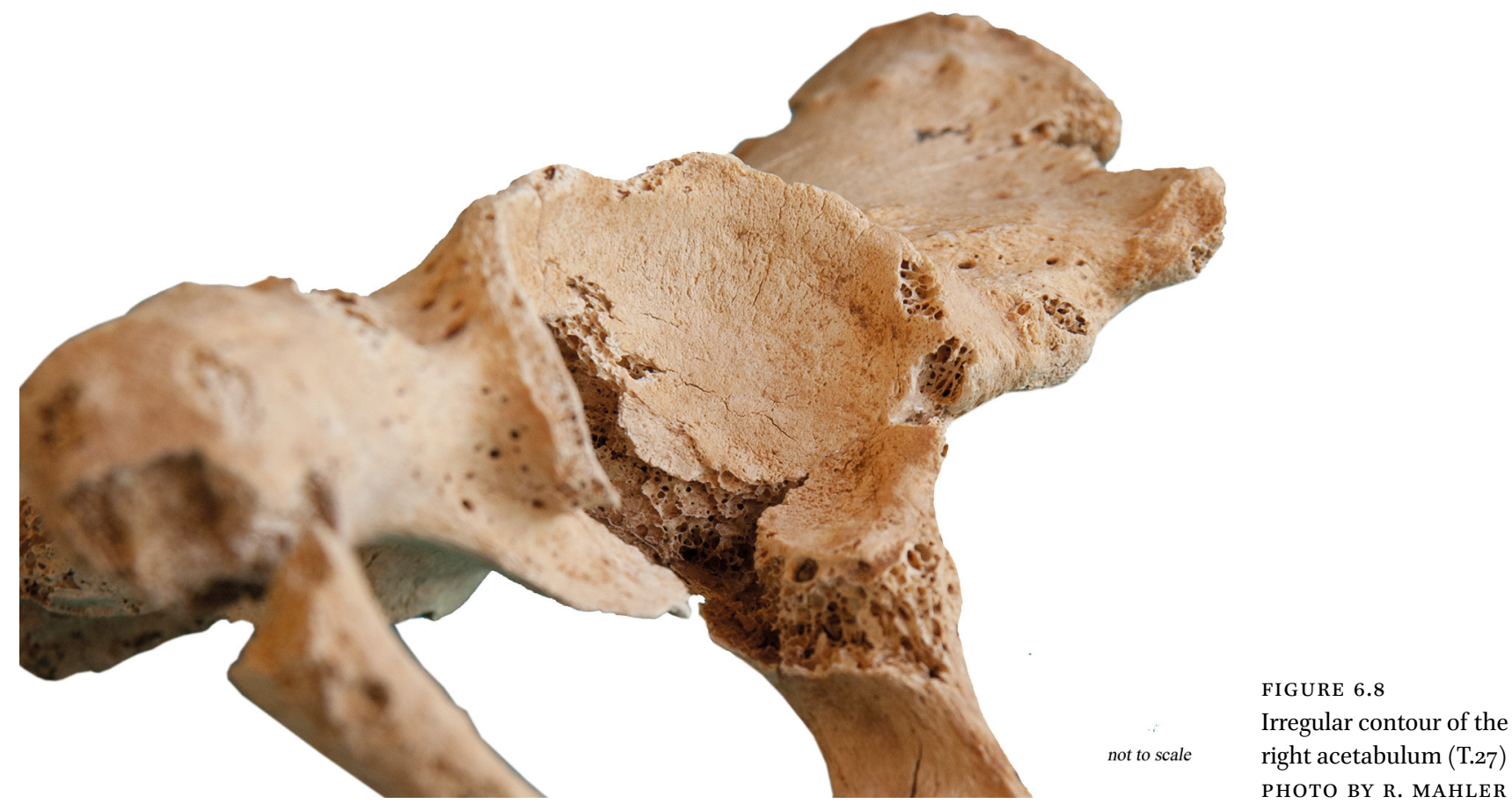

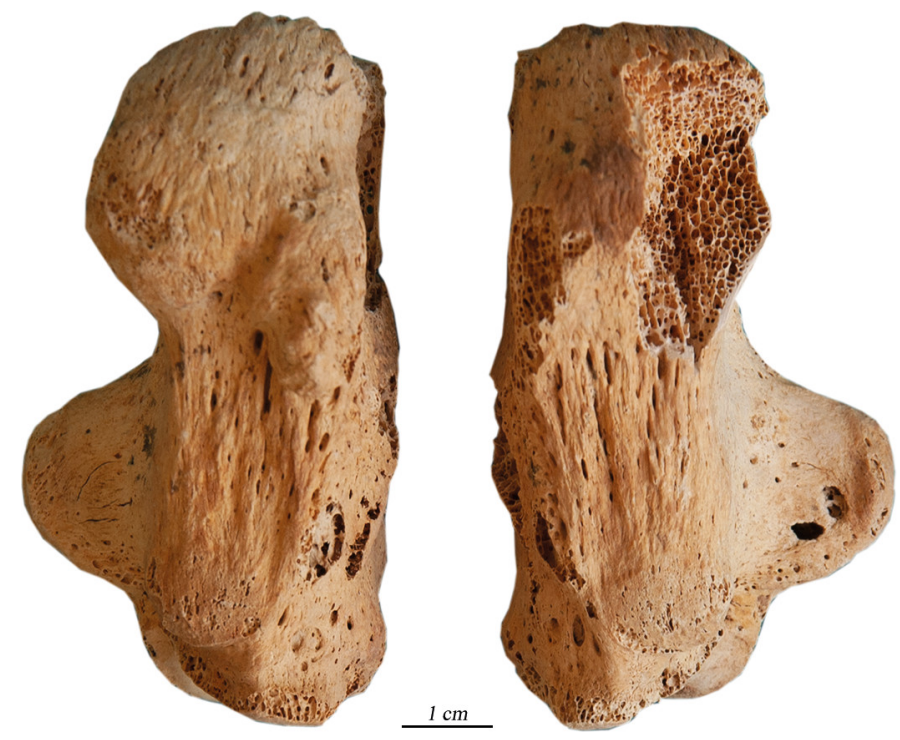

FIGURE 6.9

Overgrowth of the lateral processes of both calcanei (T.27) PHOTO BY R. MAHLER 
Tumulus 28-type III

\begin{tabular}{ll}
\hline MNI & 1 \\
Sex & F \\
Age & $40-55$ \\
Stature & 160 \\
\hline
\end{tabular}

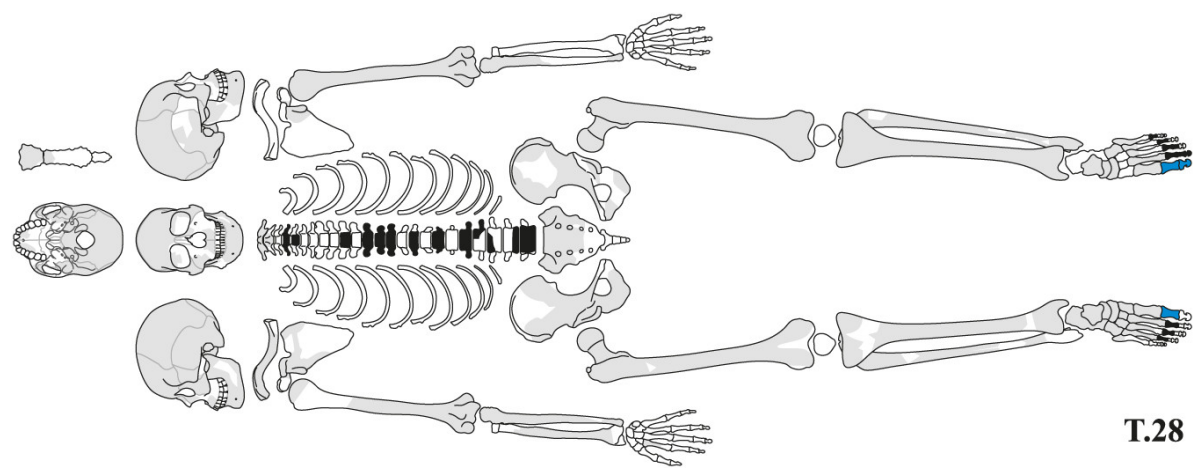

Preserved fragments of:

- 15 ribs

Length measurements (M1):

\begin{tabular}{lllllll}
\hline bone: & humerus & radius & ulna & femur & tibia & fibula \\
right: & - & - & $259(? ? ?)$ & $438(? ? ?)$ & - & - \\
left: & 315 & - & - & 439 & 375 & $369(?)$ \\
\hline
\end{tabular}

\section{Dental pathology}

1) apical granuloma at the position of teeth $42($ ?) and $43(?)$

2) abscesses at the position of teeth 47 (?), $46(?), 45(?), 44,41,31$ and 34

\section{Skeletal pathology}

DJD: 1) OA: small marginal lipping and light pitting on the surface of the left mandibular fossa

2) OA: small marginal lipping and small osteophytes on the surface of the left TMJ articular tubercle

3) OA: small marginal lipping and light pitting on the proximal joint surface of the right fibula and left tibia

4) OA: small marginal lipping and light pitting on the lunate surfaces of both acetabulae

5) OA: light-to-medium marginal lipping and light-to-moderate pitting on the joint surfaces of articular processes of the cervical vertebrae (stronger on the left)

6) OA: small marginal lipping and small osteophytes of the right inferior articular process of an upper lumbar vertebra

cribra orbitalia: 1

other: 1) light inactive inflammation of the right frontal sinus (?) and the right maxillary sinus; moderate changes in the left frontal sinus (?)

2) multiple small button osteomas on the outer table of the left parietal bone, one on the right parietal bone and three on the frontal bone

\section{Trauma}

None observed.

\section{Other observations}

None. 


\section{Appendix: Pilot Study of Paleogenomic Analysis of El-Zuma Samples}

\section{Introduction}

In February 2017, a pilot project was undertaken to determine DNA preservation in previously analysed skeletal remains excavated at El-Zuma. Bone and tooth samples were selected for screening of ancient DNA (aDNA) to determine the viability of paleogenomic research and then assemble mitochondrial genomes of successful individuals to map the genetic landscape of those interred at this site. These data would provide novel insights into the ancestry of El-Zuma individuals using mitochondrial (mt) DNA to be combined with other lines of evidence, including stable isotope analyses, anthropological evaluations, and the archaeological context of these tumuli, to contribute to a more comprehensive understanding of those living in the Dongola Reach.

\section{$2 \quad$ Samples from the El-Zuma Tumuli}

To begin this project, intact teeth and/or temporal bones were selected from the collection stored at the field house near the excavation site. Permission to sample for ancient DNA trials was granted by Assist. Prof. Mahmoud El-Tayeb, the director of the Early Makuria Research Project. Work was assisted by Robert Mahler, and in the field by Magdalena Srienc when retrieving samples. Having been previously evaluated for anthropometric traits and anthropological analyses, samples were selected by the above personnel for best results of retrieving sterile bone or tooth tissue; this included intact or uncompromised teeth and temporal bones, specifically the petrous portion. Listed in Table 6.4, the following samples were photographed (in the field) and recorded for exportation from Sudan.

As detailed above, 26 samples in total were exported, representing 16 unique individuals excavated from the El-Zuma burials; all samples were transported to the University of Zürich for processing as part of previous agreements with the project director. There, further photographs were taken of the remains before processing [Figs. 6.10-6.15]. Samples will be returned for permanent storage at the field site.

\section{Sample Processing and Extraction Methods}

Method optimization was a parallel objective to the evaluation of DNA viability for the samples from the El-Zuma archaeological site. Therefore, the samples were selected for and subjected to multiple state-of-the-art methods to retrieve ancient genetic material. A subset of samples was selected to trial multiple protocols to retrieve ancient DNA for next-generation sequencing (NGS), before applying a successful technique to the rest of the sample cohort. While being mostly or fully intact, the bone and tooth samples from El-Zuma had noticeable diagenic issues. In general, most bone tissue is slightly to moderately bleached or discoloured/stained from the soil, moderately to extensively weathered (with respect to the external cortical bone), and the tissue integrity is soft, suggesting the mineral component is compromised. For some skeletal remains, the presence of mould (i.e. extensive bioerosion) was noted and attests to the unfavourable conditions that may have damaged the tissue, as detailed further in this chapter (Kendall et al. 2018). For the teeth, the enamel is in good condition and any calculus is also intact. However, cementum is flaky or brittle and the dentine is bleached much like the bone tissue, but more severely, and can be soft, which again hints that demineralization may be a concern. Below are examples of the damage sustained by the skeletal remains from the tumuli at El-Zuma [Fig. 6.16].

For the samples from El-Zuma, the depositional environment - namely hot, humid, open-air, underground tumuli-likely caused the observed diagenic changes for both the teeth and bones mentioned above, in addition to looting (Mahmoud El-Tayeb, Skowrońska, and Czyżewska 2016; Mahmoud El-Tayeb 2005). Environment has a large effect on the preservation of any endogenous DNA, and is especially the case for those from arid climates (Poinar 2003; Hansen et al. 2017; Pinhasi et al. 2015; Allentoft et al. 2012). Despite the preservation issues, this pilot project was undertaken since these paleogenomic data stand to add another line of evidence to understand differences observed in the archaeological context. Aggressive methods were selected to be used to retrieve genetic material from these seemingly compromised substrates (i.e. bones and teeth). In addition to environmental contamination, remains had been physically handled for many years by researchers during and after excavation (as all remains are), and it was assumed contamination (i.e. presence of modern DNA) would be an obstacle to be 
TABLE 6.4 List of El-Zuma samples exported from Sudan and currently in storage at the University of Zürich

\begin{tabular}{|c|c|c|c|c|c|c|}
\hline & $\begin{array}{l}\text { Date } \\
\text { collected }\end{array}$ & $\begin{array}{l}\text { Tumulus } \\
\text { no. }\end{array}$ & $\begin{array}{l}\text { Sample } \\
\text { type }\end{array}$ & Sample & $\begin{array}{l}\text { Retrieval method/ } \\
\text { state of remains }\end{array}$ & Field notes and other observations \\
\hline 1 & $27 / \mathrm{Feb} / 18$ & $\mathrm{~T} .8$ & bone & R Temporal & broken & \\
\hline 2 & $27 / \mathrm{Feb} / 18$ & T.10 & bone & L Petrous portion & broken & \\
\hline 3 & $27 / \mathrm{Feb} / 18$ & T.10 & bone & R Temporal & broken & \\
\hline 4 & $27 / \mathrm{Feb} / 18$ & T.11 & bone & R Temporal & broken & \\
\hline 5 & $27 / \mathrm{Feb} / 18$ & T.11 & bone & L Petrous portion & broken & \\
\hline 6 & $27 / \mathrm{Feb} / 18$ & T.14 & bone & R Temporal & broken & glued/reconstruction \\
\hline 7 & $27 / \mathrm{Feb} / 18$ & T.15 & tooth & LPM2 (maxillary) & extracted & \\
\hline 8 & $27 / \mathrm{Feb} / 18$ & T.15 & tooth & LC1 (mandibular) & extracted & \\
\hline 9 & $27 / \mathrm{Feb} / 18$ & T.15 & bone & L Petrous portion & broken & very weathered, a bit mouldy \\
\hline 10 & $27 / \mathrm{Feb} / 18$ & T.16 & bone & R Petrous portion & broken & some glue/reconstruction \\
\hline 11 & $27 / \mathrm{Feb} / 18$ & T.17 & bone & L Temporal & broken & some lesions on ventral slope of petrous \\
\hline 12 & $27 / \mathrm{Feb} / 18$ & T.18 & bone & L Temporal & broken & very weathered \\
\hline 13 & $27 / \mathrm{Feb} / 18$ & T.18 & bone & R Temporal & excised & very weathered \\
\hline 14 & $27 / \mathrm{Feb} / 18$ & T.19 & bone & R Petrous portion & broken & \\
\hline 15 & $27 / \mathrm{Feb} / 18$ & T.20 & bone & L Petrous portion & excised & some glue/reconstruction, mouldy \\
\hline 16 & $27 / \mathrm{Feb} / 18$ & T.2O & bone & R Petrous portion & excised & some glue/reconstruction, mouldy \\
\hline 17 & $27 / \mathrm{Feb} / 18$ & T.22 & bone & L Temporal & excised & some glue/reconstruction \\
\hline 18 & $27 / \mathrm{Feb} / 18$ & T.24 & bone & R Petrous portion & broken & \\
\hline 19 & $27 / \mathrm{Feb} / 18$ & T.25 & bone & R Petrous portion & broken & \\
\hline 20 & $27 / \mathrm{Feb} / 18$ & T.25 & bone & L Petrous portion & broken & \\
\hline 21 & $27 / \mathrm{Feb} / 18$ & T.25 & tooth & RPM1 (maxillary) & broken & \\
\hline 22 & $27 / \mathrm{Feb} / 18$ & T.25 & tooth & RPM2 (maxillary) & broken & \\
\hline 23 & $27 / \mathrm{Feb} / 18$ & T.26 & bone & L Petrous portion & broken & some glue/reconstruction \\
\hline 24 & $27 / \mathrm{Feb} / 18$ & T.27 & tooth & LPM2 (maxillary) & extracted & \\
\hline 25 & $27 / \mathrm{Feb} / 18$ & T.27 & tooth & RPM2 (maxillary) & extracted & \\
\hline 26 & $27 / \mathrm{Feb} / 18$ & T.28 & bone & R Petrous portion & broken & \\
\hline
\end{tabular}

Abbreviations used: $\mathrm{T}$ = Tumulus, $\mathrm{R}=$ right side, $\mathrm{L}=$ left side, $\mathrm{C}=$ canine, $\mathrm{PM}=$ premolar PRODUCED BY A. BREIDENSTEIN
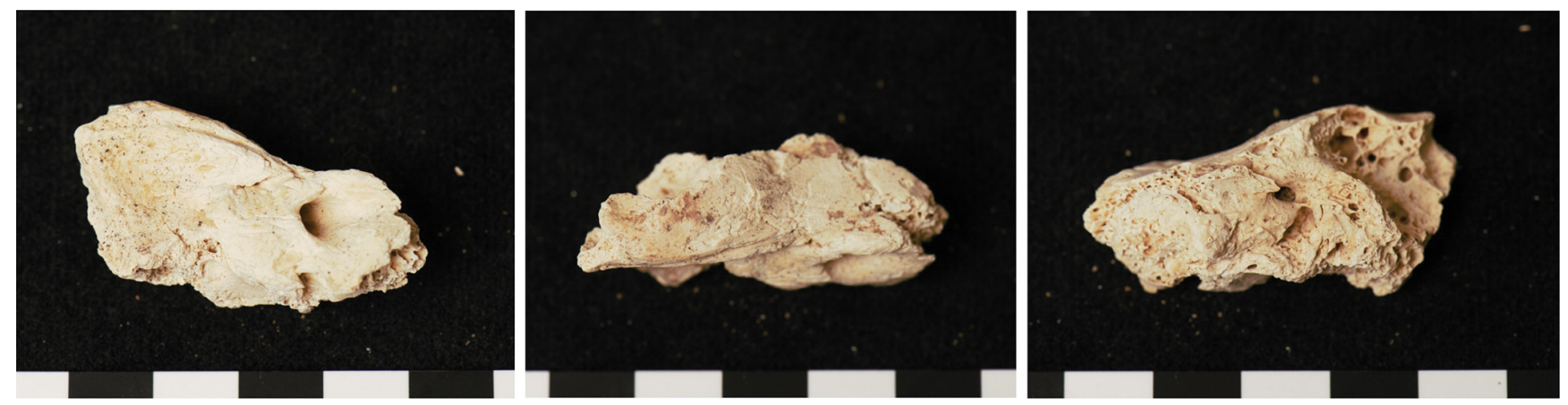

FIGURE 6.10 El-Zuma Tumulus 10 sample-left petrous pyramid of temporal bone. Left: dorsal view. Middle: inferior view. Right: ventral view PHOTOS BY A. BREIDENSTEIN 

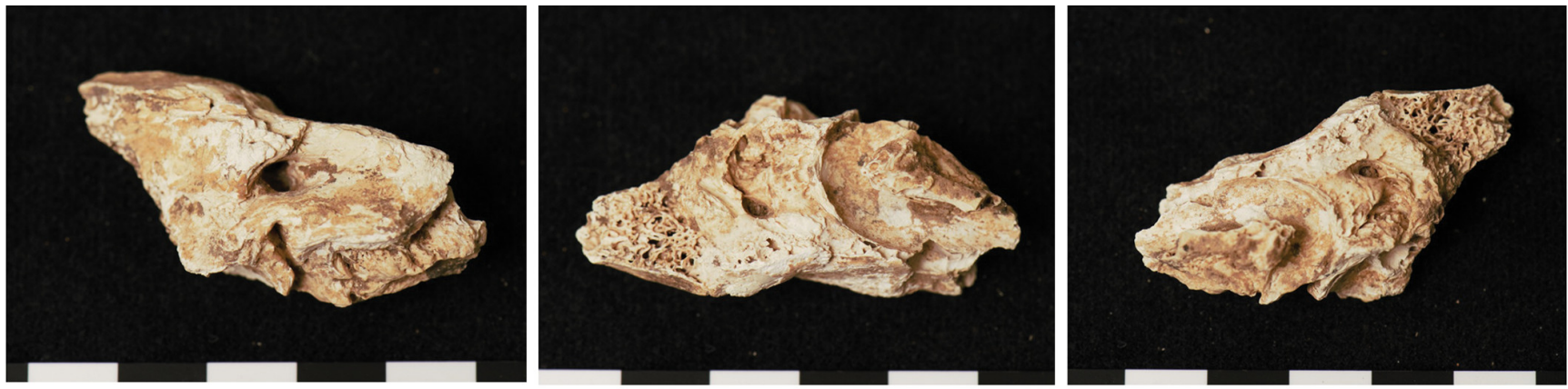

FIGURE 6.11 El-Zuma Tumulus 11 sample-left petrous pyramid of temporal bone. Left: dorsal view.

Middle: inferior view. Right: ventral view PHOTOS BY A. BREIDENSTEIN
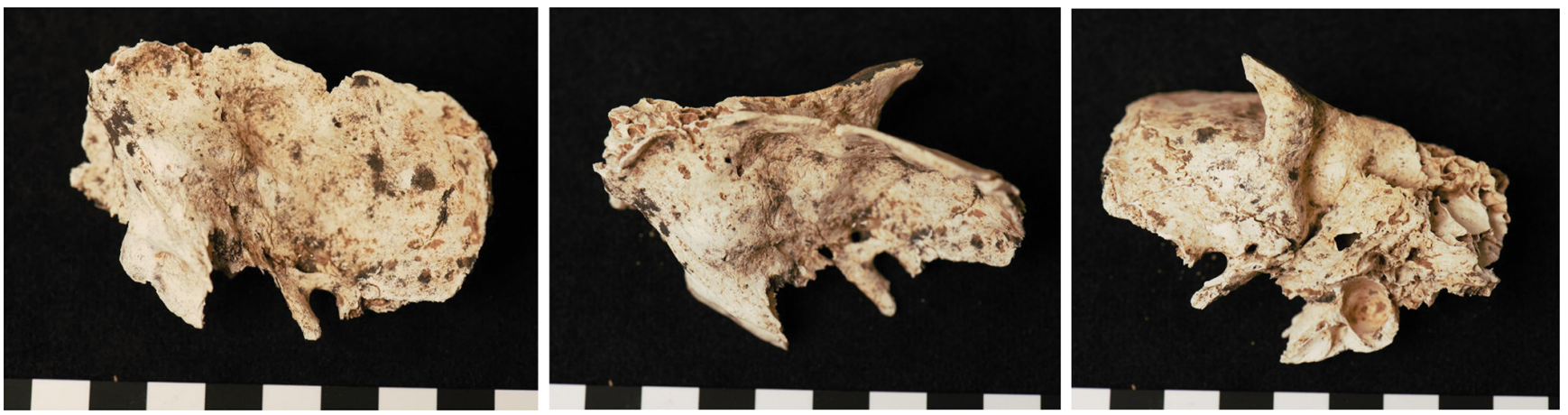

FIGURE 6.12 El-Zuma Tumulus 15 sample-left temporal bone. Left: medial view. Middle: superior view.

Right: inferior view

PHOTOS BY A. BREIDENSTEIN
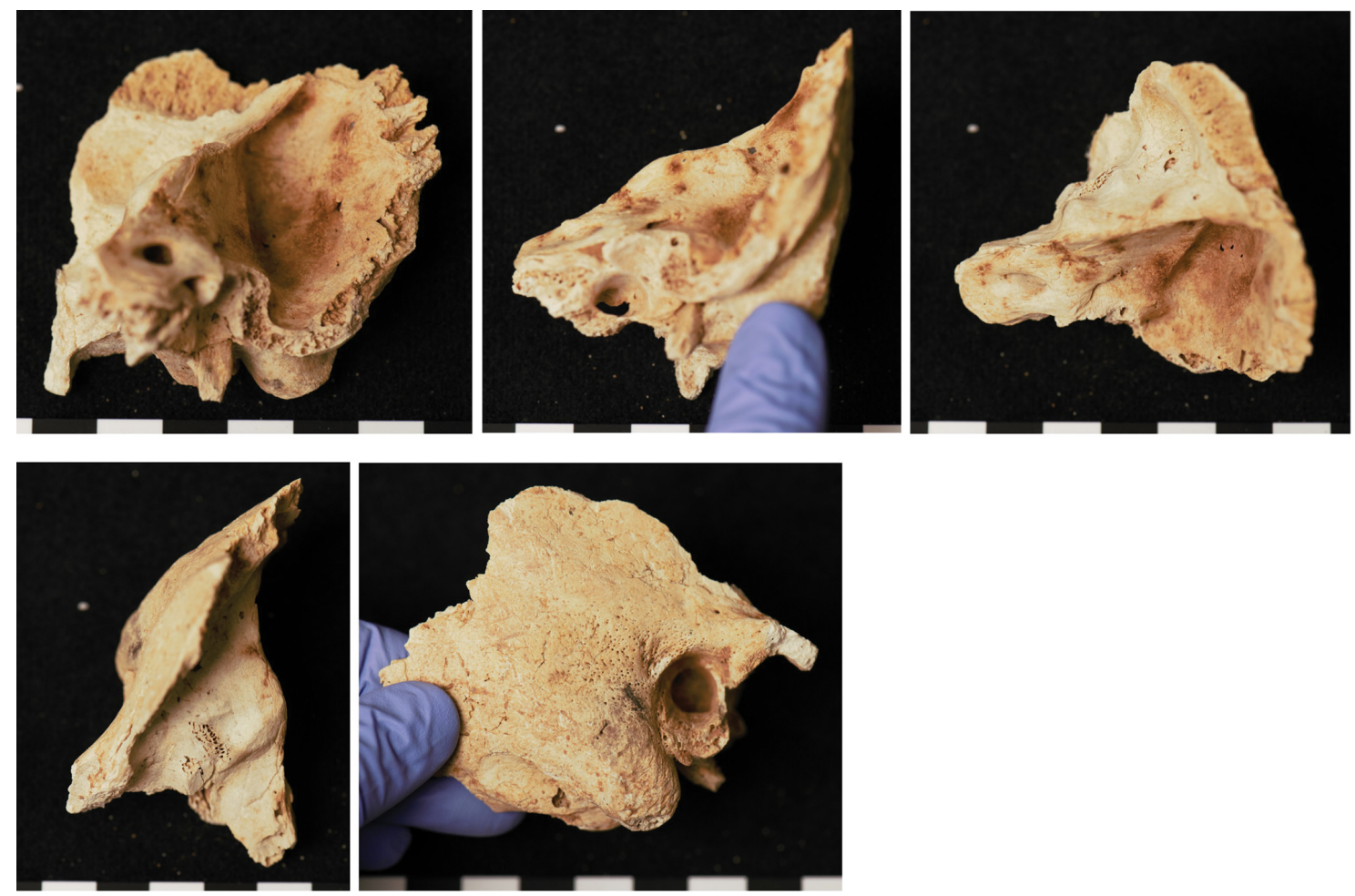

FIGURE 6.13 El-Zuma Tumulus 17 sample-left temporal bone. Left upper: medial view. Middle upper: ventral view. Right upper: superior view. Left lower: ventral view. Right lower: lateral view PHOTOS BY A. BREIDENSTEIN 

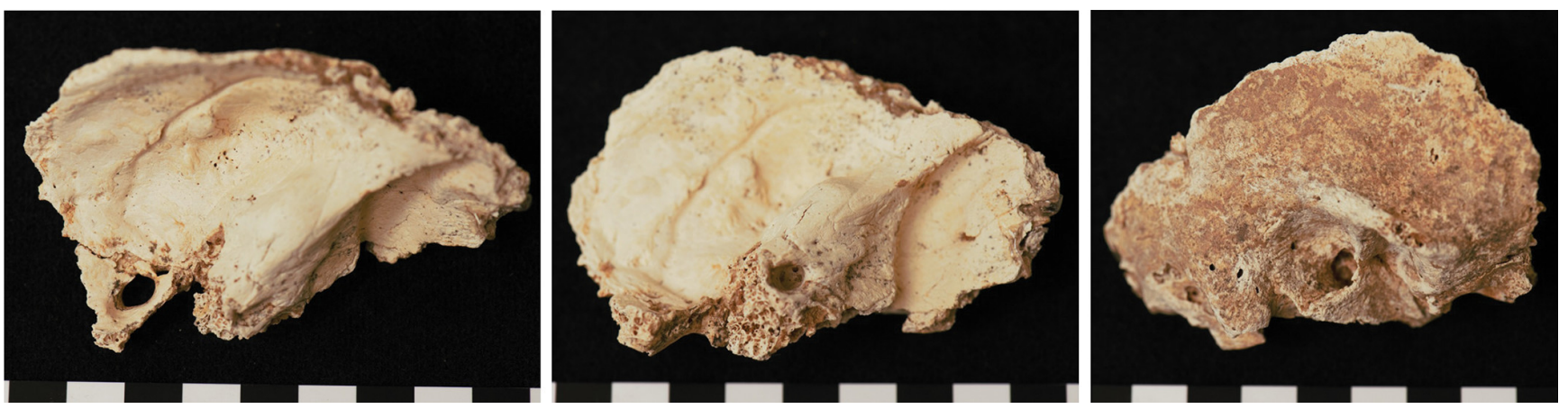

FIGURE 6.14 El-Zuma Tumulus 18 sample—right temporal bone. Left: superior view. Middle: medial

view. Right: lateral view

PHOTOS BY A. BREIDENSTEIN
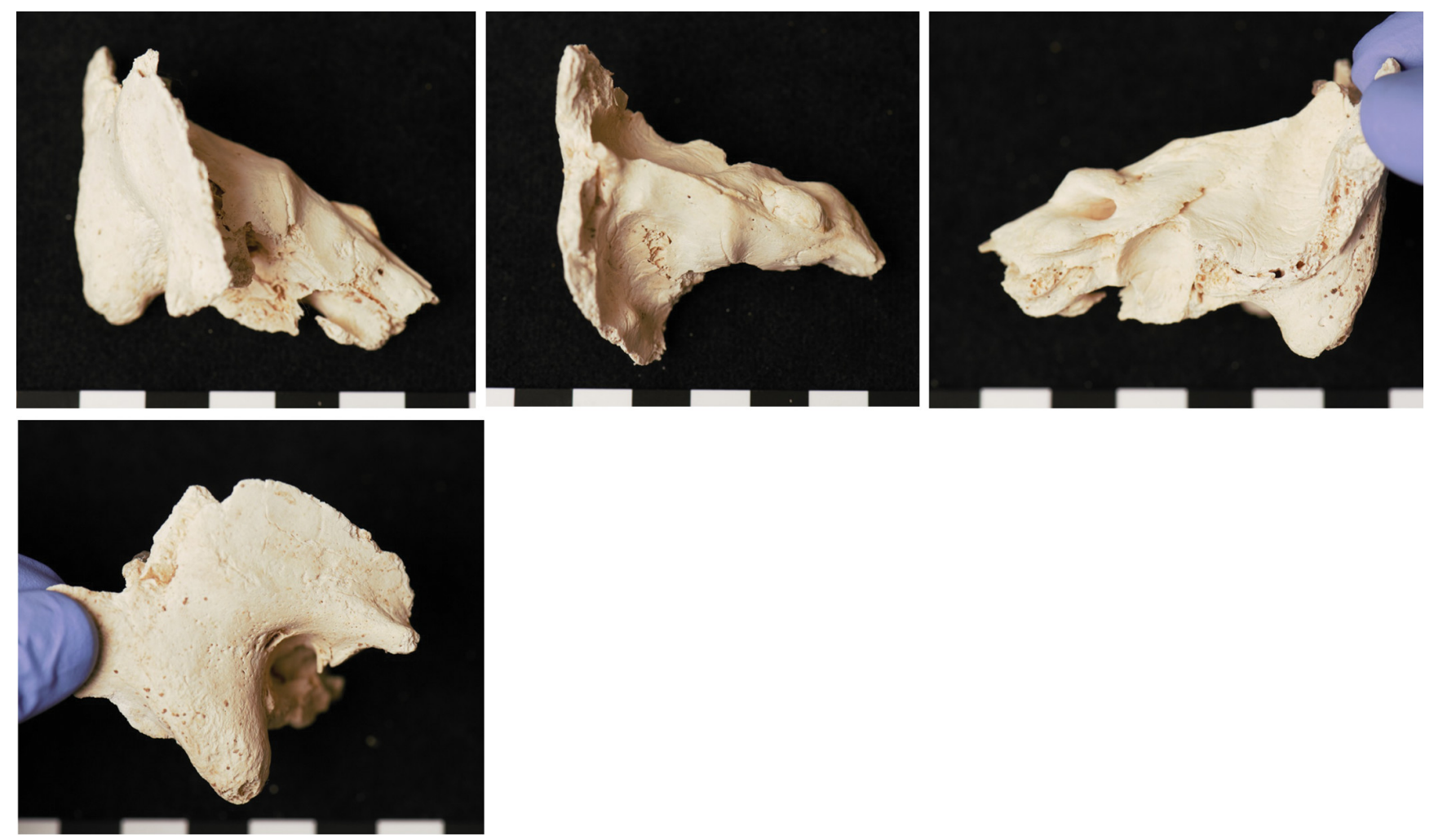

FIGURE 6.15 El-Zuma Tumulus 28 sample—right petrous pyramid of temporal bone. Left upper: dorsal view. Middle upper: superior view. Right upper: ventral view. Left lower: lateral view PHOTOS BY A. BREIDENSTEIN

addressed through processing methodologies. To mitigate the above issues, three methodologies were trialled to compare the yield of ancient DNA across the selected protocols using a subset of six bone samples [Table 6.5] to test these methods. Additionally, several samples were processed for DNA extraction but have yet to be extracted due to time constraints (i.e. tooth samples, not listed).

To begin, samples were processed according to the most current protocols optimized for materials from Africa to obtain sterile tissue from the remains (Gamba et al. 2014;
Pinhasi et al. 2015; Gallego Llorente et al. 2015; Damgaard et al. 2015; Hansen et al. 2017), in addition to more experimental methods to mitigate high contamination or compromised tissues, as outlined in Gondek, Boessenkool, and Star (2018). The first subset of samples was chosen for multiple methodologies to evaluate DNA yield and optimize extraction methods to be applied to the El-Zuma samples following these trials [Table 6.5].

Temporal bone samples were first decontaminated in a Uv-crosslinker (at least 15 min each side) before mechanically removing a layer of the outer cortical bone (i.e. most 

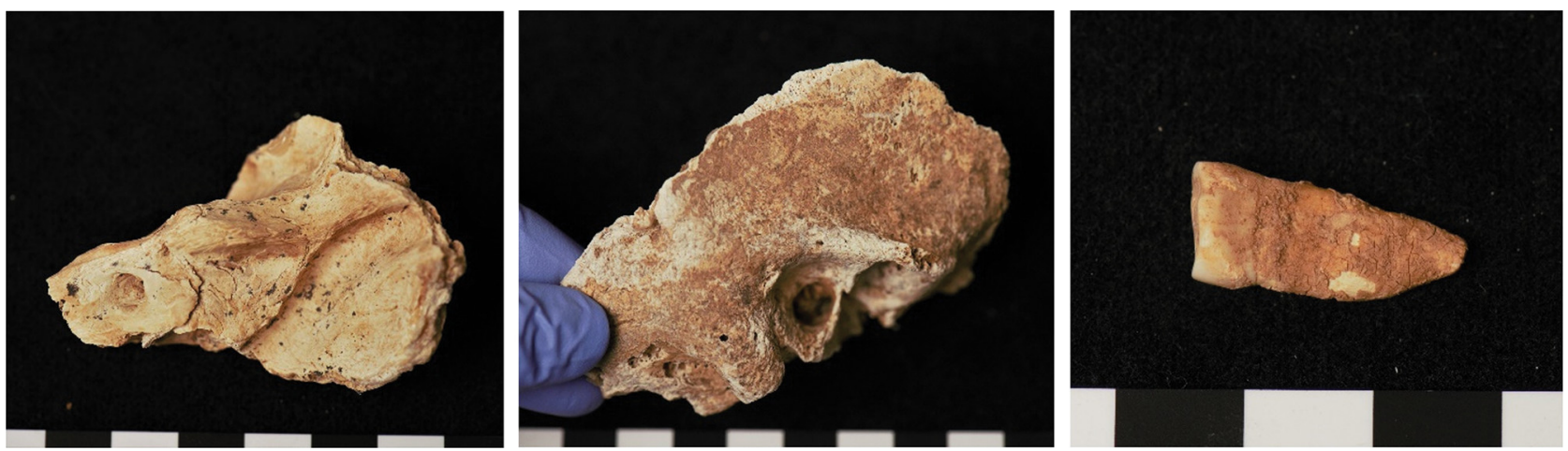

FIGURE 6.16

Examples of taphonomic damage to skeletal remains and teeth found at El-Zuma. Left: right petrous from Tumulus 16 showing mould growth on external cortical bone. Middle: right temporal from Tumulus 18 showing extensive weathering on external surface. Right: right 2nd premolar from Tumulus 27 showing flaky or brittle cementum found on some teeth

PHOTOS BY A. BREIDENSTEIN

TABLE 6.5 El-Zuma samples prepped for DNA extraction and notes about the state of the remains before processing; two aliquots were obtained for double digestion protocols. Additional aliquots for ZUM18 were taken for a bleach pre-treatment before double digestion, designated as " $\mathrm{B}$ "

\begin{tabular}{|c|c|c|c|c|c|}
\hline $\begin{array}{l}\text { Sample } \\
\text { name }\end{array}$ & Sample & $\begin{array}{l}\text { Tissue } \\
\text { type }\end{array}$ & $\begin{array}{l}\text { Processing } \\
\text { method }\end{array}$ & $\begin{array}{l}\text { Sample amount } \\
\text { (+remaining) }\end{array}$ & Sampling / lab notes \\
\hline ZUM1O & L Petrous & Bone & crush & $2 \times 100 \mathrm{mg}+213 \mathrm{mg}$ & $\begin{array}{l}\text { very soft, weathered, chalky bone, flaky when } \\
\text { crushed }\end{array}$ \\
\hline ZUM11 & L Petrous & Bone & crush & $2 \times 100 \mathrm{mg}+913 \mathrm{mg}$ & $\begin{array}{l}\text { very soft, weathered, some external and internal } \\
\text { mould, darker stains }\end{array}$ \\
\hline ZUM15 & L Petrous & Bone & crush & $2 \times 200 \mathrm{mg}+47 \mathrm{omg}$ & $\begin{array}{l}\text { weathered, chalky, mouldy, powdered better than } \\
\text { expected }\end{array}$ \\
\hline ZUM17 & L Petrous & Bone & crush & $2 \times 200 \mathrm{mg}+1410 \mathrm{mg}$ & (none) \\
\hline ZUM18 & R Petrous & Bone & crush & $2 \times 200 \mathrm{mg}+370 \mathrm{mg}$ & $\begin{array}{l}\text { mouldy, chalky, soft, but produced a fine powder, } \\
\text { superficial surface removed }\end{array}$ \\
\hline ZUM18_B & R Petrous & Bone & crush & $2 \times 200 \mathrm{mg}$ & $\begin{array}{l}\text { (see above, additional powder for bleach treat- } \\
\text { ment method) }\end{array}$ \\
\hline ZUM28 & R Petrous & Bone & crush & $2 \times 100 \mathrm{mg}+201 \mathrm{mg}$ & $\begin{array}{l}\text { soft bone, very bleached in colour, maybe osteo- } \\
\text { porotic; some glue }\end{array}$ \\
\hline
\end{tabular}

Abbreviations: $\mathrm{R}=$ right, $\mathrm{L}=$ left, $\mathrm{mg}=$ milligrams

PRODUCED BY A. BREIDENSTEIN

likely to be contaminated), following another round of UV decontamination. Bone samples were then powdered into multiple aliquots using a stainless steel mortar and pestle (Gondek, Boessenkool, and Star 2018), then subjected to various pre-treatments before DNA extraction, for a total of ten trials: three were subjected to a standard digestion ("SD") method (detailed in Schuenemann et al. 2017, with modification from Dabney et al. 2013), six to a pre-digestion step before the standard extraction, known as a double digest ("DD") (modelled from Damgaard et al.
2015 and Schroeder, de Barros Damgaard, and Allentoft 2019), and one with an experimental bleach pre-treatment before a double digestion of the powder ("B") (Boessenkool et al. 2017; Kemp and Smith 2005). Samples were processed in two labs while trialling new methodologies: the Institute of Evolutionary Medicine, University of Zürich, Switzerland (UZH) and the Centre for Ecological and Evolutionary Synthesis, University of Oslo, Norway (UiO). DNA extracts were built into double-stranded libraries as outlined in Meyer and Kircher (2010), then 
TABLE 6.6 Shotgun sequencing results for the subset of El-Zuma samples using three protocols with two different pre-treatments, while standard methodology is single digestion "SD". Samples were treated with a pre-digestion ("DD") and/or bleach ("B") to mitigate modern or external contamination. Cluster factor is a measurement of sequence uniqueness within the libraries

\begin{tabular}{lllllllll}
\hline Sample name & $\begin{array}{l}\text { Extraction } \\
\text { method }\end{array}$ & $\begin{array}{l}\text { Mapped } \\
\text { human } \\
\text { reads }\end{array}$ & $\begin{array}{l}\text { Endogenous } \\
\text { DNA (\%) }\end{array}$ & $\begin{array}{l}\text { Cluster } \\
\text { factor }\end{array}$ & $\begin{array}{l}\text { Mapped } \\
\text { MT reads }\end{array}$ & $\begin{array}{l}\text { Avg. MT } \\
\text { coverage (X) }\end{array}$ & $\begin{array}{l}\text { MT/NUC } \\
\text { ratio }\end{array}$ & $\begin{array}{l}\text { Avg. fragment } \\
\text { length (bp) }\end{array}$ \\
\hline ZUM10_DD & DD & 134 & 0.011 & 1.067 & 0 & NaN & 0 & 54.31 \\
ZUM10_SD & SD & 201 & 0.011 & 1.025 & 0 & NaN & 0 & 38.29 \\
ZUM11_DD & DD & 121 & 0.008 & 1.058 & 0 & NaN & 0 & 55.88 \\
ZUM11_SD & SD & 259 & 0.014 & 1.058 & 0 & NaN & 0 & 42.12 \\
ZUM15_oslo & DD & 222 & 0.015 & 1.032 & 0 & NaN & 0 & 51.29 \\
ZUM17_oslo & DD & 276 & 0.014 & 1.033 & 0 & NaN & 0 & 57.34 \\
ZUM18_oslo & DD & 106 & 0.011 & 1.075 & 0 & NaN & 0 & 48.36 \\
ZUM18B_oslo & B+DD & 8188 & 0.215 & 1.345 & 11 & 0.042126 & 244.91 & 65.12 \\
ZUM28_DD & DD & 650 & 0.134 & 1.318 & 1 & 0.002655 & 168.5 & 75.13 \\
ZUM28_SD & SD & 3554 & 0.063 & 1.301 & 5 & 0.022451 & 290.21 & 67.49 \\
\hline
\end{tabular}

"oslo" = samples processed, and libraries constructed at UiO. "X" = measure of coverage of the genome where the number designates the average number of unique reads covering any location along the genome. "MT" = mitochondrial DNA, "NUC" = nuclear DNA, bp = base pairs. "NaN" = no sequence reads available

PRODUCED BY A. BREIDENSTEIN

dual-indexed according to Kircher, Sawyer, and Meyer (2012). Libraries were 75 paired-end sequenced on an Illumina HiSeq4ooo platform at the UiO and/or the Functional Genomics Center Zürich (at the UZH). Raw data reads were pre-processed bioinformatically, then mapped against a human reference genome using the EAGER program, a pipeline optimized for degraded sequence data (Peltzer et al. 2016). For those DNA extracts with obtained human mitochondrial DNA from shotgun sequencing and evidence of the material being ancient, magnetic bead hybridization was applied to enrich and concentrate for mtDNA, as detailed in Maricic, Whitten, and Pääbo (2010), then sequenced for mitogenome assembly and analysis.

\section{DNA Extraction and Sequencing Results with Discussion}

Despite employing mechanical and/or chemical means of mitigating contamination and the use of NGS strategies to obtain ancient genetic material, only one of the trials was successful in retrieving human DNA characterized as ancient. Shotgun sequencing yields ranged from around 100 to over 8,ooo reads (or molecules) mapping to the human reference genome and endogenous contents (i.e. amount of authentic human DNA) were between 0.01 and
$0.2 \%$, which were both disappointing outcomes. Results are listed in Table 6.6. Moreover, mitochondrial reads were only obtained in three trials, one of which was with the bleach treatment, but at an unappreciable level (i.e. between 1 and 11 reads).

For most El-Zuma samples listed above, two libraries were built from separate extracts subjected to different extraction methods (e.g. single digest, double digest). These sequenced libraries are theoretically the same individual and could thus be merged post-sequencing to evaluate authenticity criteria (i.e. mapped human reads, damage patterns, fragment length). After merging, the resulting metrics are listed in Table 6.7.

As expected, merging these data increased mapped reads - between a few hundred to more than 9,0oo, while the endogenous contents were averaged. These merged read files are also more useful to evaluate damage patterns. Ancient or degraded DNA can be characterized by an accumulation of misincorporation lesions at the terminal ends of sequences, and these frequencies can be plotted along the length of the sequence reads to create a "damage profile" (Briggs et al. 2007; Dabney, Meyer, and Pääbo 2013; Krause et al. 2010). These patterns function as an authentication criterion when working with aDNA and frequencies typically reach percentages of at least 10 and up to 40 (Dabney, Meyer, and Pääbo 2013; Krause et al. 2010). For the El-Zuma extracts, damage frequencies were 
TABLE 6.7 Merged shotgun sequence data per individual

\begin{tabular}{|c|c|c|c|c|c|c|c|c|c|c|}
\hline $\begin{array}{l}\text { Sample } \\
\text { name }\end{array}$ & $\begin{array}{l}\text { Mapped } \\
\text { human } \\
\text { reads }\end{array}$ & $\begin{array}{l}\text { Endogen- } \\
\text { ous DNA } \\
(\%)\end{array}$ & $\begin{array}{l}\text { Cluster } \\
\text { factor }\end{array}$ & $\begin{array}{l}\text { Mean NUC } \\
\text { coverage } \\
(\mathrm{X})\end{array}$ & $\begin{array}{l}\text { Mapped } \\
\text { MT reads }\end{array}$ & $\begin{array}{l}\text { Avg. MT } \\
\text { coverage } \\
(\mathrm{X})\end{array}$ & $\begin{array}{l}\mathrm{MT} / \mathrm{NUC} \\
\text { ratio }\end{array}$ & $\begin{array}{l}\text { DMG 1st } \\
\text { base } 5^{\prime}(\%)\end{array}$ & $\begin{array}{l}\text { DMG 1st } \\
\text { base } 3^{\prime}(\%)\end{array}$ & $\begin{array}{l}\text { Avg. fragment } \\
\text { length (bp) }\end{array}$ \\
\hline ZUM1O & 333 & 0.011 & 1.048 & $\mathrm{O}$ & o & $\mathrm{NaN}$ & $\mathrm{O}$ & o.0196 & 0.0465 & 44.61 \\
\hline ZUM11 & 378 & 0.011 & 1.063 & $\mathrm{O}$ & $\mathrm{O}$ & $\mathrm{NaN}$ & $\mathrm{O}$ & o.0333 & 0.0244 & 46.34 \\
\hline ZUM15 & 1772 & 0.017 & $1.15^{8}$ & O & $\mathrm{O}$ & $\mathrm{NaN}$ & $\mathrm{O}$ & O.0533 & 0.057 & $49 \cdot 74$ \\
\hline ZUM17 & 2783 & 0.016 & 1.349 & $\mathrm{O}$ & $\mathrm{O}$ & $\mathrm{NaN}$ & $\mathrm{O}$ & 0.0142 & 0.0169 & 48.82 \\
\hline ZUM1 8 & 9477 & 0.07 & 1.32 & 0.0002 & 11 & 0.0421 & 222.95 & 0.0127 & 0.0153 & 61.79 \\
\hline ZUM28 & 4201 & O.068 & 1.305 & 0.0001 & 6 & 0.0251 & 269.83 & 0.0085 & 0.0069 & 68.67 \\
\hline
\end{tabular}

"NUC" = nuclear DNA, "MT" = mitochondrial DNA, "DMG" = damage, "bp" = base pairs, "NaN" = no sequences available

PRODUCED BY A. BREIDENSTEIN

TABLE 6.8 Mapped mitochondrial reads for samples subjected to enrichment

\begin{tabular}{|c|c|c|c|c|c|c|c|c|}
\hline Sample name & $\begin{array}{l}\text { Mapped } \\
\text { мт human } \\
\text { reads }\end{array}$ & $\begin{array}{l}\text { Endo- } \\
\text { genous } \\
\text { DNA (\%) }\end{array}$ & $\begin{array}{l}\text { Cluster } \\
\text { factor }\end{array}$ & $\begin{array}{l}\text { Mapped } \\
\text { MT reads }\end{array}$ & $\begin{array}{l}\text { Avg. MT } \\
\text { coverage } \\
(X)\end{array}$ & $\begin{array}{l}\text { Damage at } \\
\text { 1st base } 5^{\prime}\end{array}$ & $\begin{array}{l}\text { Damage at } \\
\text { 1st base } 3^{\prime}\end{array}$ & $\begin{array}{l}\text { Avg. fragment } \\
\text { length (bp) }\end{array}$ \\
\hline ZUM18B_oslo & 493 & $1.8 \circ 8$ & 265.022 & 493 & 2.0538 & $\mathrm{o}$ & 0.0079 & 69.02 \\
\hline ZUM28_DD & 7 & 0.347 & 396 & 7 & 0.0179 & $\mathrm{O}$ & $\mathrm{O}$ & 42.29 \\
\hline ZUM28_SD & 80 & 0.222 & 174.488 & 80 & 0.4021 & $\mathrm{O}$ & $\mathrm{O}$ & 83.28 \\
\hline
\end{tabular}

"MT" = mitochondrial, "bp" = base pairs, "oslo" = prepped at UiO, "DD" = double digest, "SD" = single digest

PRODUCED BY A. BREIDENSTEIN

at most $5 \%$, and most plots did not demonstrate a pattern that would indicate the genetic material to be ancient. One sample, ZUM15, showed some hints of a characteristic pattern, and with short fragment sizes $(30-50 \mathrm{bp}$, on average), but remains unconvincing and no mitochondrial reads were obtained [Fig. 6.17]. Other damage plots for merged shotgun reads for the six individuals are found at the end of this appendix [Figs 6.19-6.23].

For all extracts in general, the mapped human reads were very low as typical read numbers should be in the range of millions, not hundreds or thousands. Additionally, the reads did not show typical characteristics to firmly indicate the obtained material was ancient, namely a lack of chemical damage patterns at the terminal ends and, in some cases (ZUM18, zUM28), fragments are too long (Briggs et al. 2007; Poinar et al. 2006). For those samples with optimal fragment lengths (i.e. less than 5 obp; zUM1o, ZUM11, ZUM15, ZUM17), the damage patterns are not ideal and, most notably, no mitochondrial reads were obtained. While not a complete metric of the sequences captured, shotgun sequencing is a snapshot of what is available in an extract; thus, zero mitochondrial reads suggests that these samples are not good candidates for the hybridization step to enrich for this genomic marker specifically.

Despite lacking any criteria for the sequences being authentically ancient, there were a few mitochondrial DNA reads obtained from three of the library trials: ZUM18B_oslo, ZUM28_DD, ZUM28_SD. These samples also had the highest number of mapped reads for the samples from the El-Zuma tumuli. Thus, these extracts were subjected to mitochondrial enrichment via hybridization with baits constructed from human mtDNA, as per Maricic, Whitten, and Pääbo (2010). These enriched extracts were amplified, pooled, and sequenced on an Illumina MiSeq platform at the Functional Genomic Center Zürich (at UZH). Sequencing results are listed in Table 6.8.

These results show that even with enrichment, the mitochondrial sequences did not increase to an appreciable level for analysis or mitogenome reconstruction. Furthermore, there are no indicators these sequences are ancient, as reads lack damage at terminal ends of the 


\section{5 ' end}

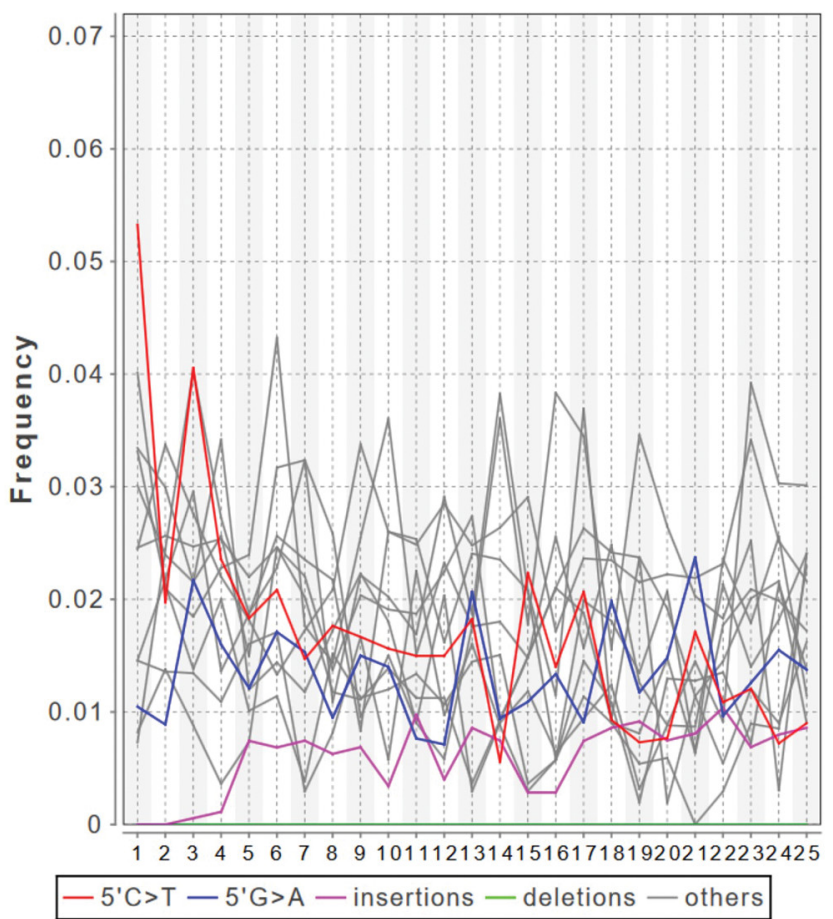

3' end

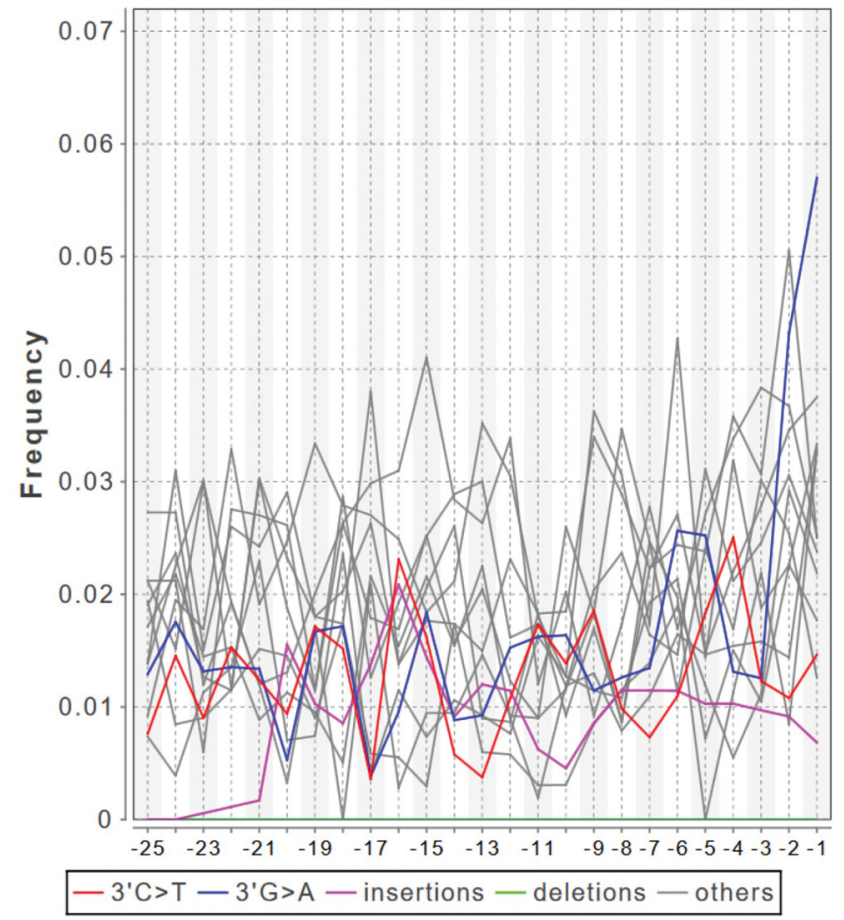

FIGURE 6.17 Merged shotgun reads for sample ZUM15 (total of 1772 reads used) showing over $5 \%$ damage accumulation at the $5^{\prime}$ and $3^{\prime}$ ends, and a characteristic "smile" profile, where $\mathrm{C}$ to $\mathrm{T}$ substitutions are higher at the $5^{\prime}$ end and $\mathrm{G}$ to A substitutions are higher at the $3^{\prime}$ end PLOT PRODUCED BY MAPDAMAGE [GINOLHAC ET AL. 2O11]

sequences and fragments are too long [Fig. 6.18]. While there were relatively very few reads to construct a damage plot, the characteristic misincorporations were not present or less than $1 \%$ [Fig. 6.18].

\section{Conclusions}

Despite cutting-edge protocols and robust measures to mitigate contamination, no ancient DNA was obtained for further analysis from ten trials using samples from the ElZuma tumuli. Taken together, these results show that DNA is likely not preserved well enough in the El-Zuma samples for paleogenomic analyses with the performed methodologies, likely due to the depositional environment of the skeletal remains in tumulus burials at this site. While these results are discouraging for further invasive analyses, tooth samples still need to be trialled and more sensitive methods, for example, single-stranded library preparation techniques (Gansauge and Meyer 2019), may be considered to obtain DNA from these samples.
These data were collected and analysed as part of a larger project, "Paleogenomic Investigation of Ancient Nubian Populations in the Past 3, ooo Years," funded by the National Geographic Society EC-224R-18, GR-000039064.

\section{By Abagail Breidenstein, PhD}

Postdoctoral Research Assistant, Institute of Evolutionary Medicine, University of Zürich 
5' end



3' end

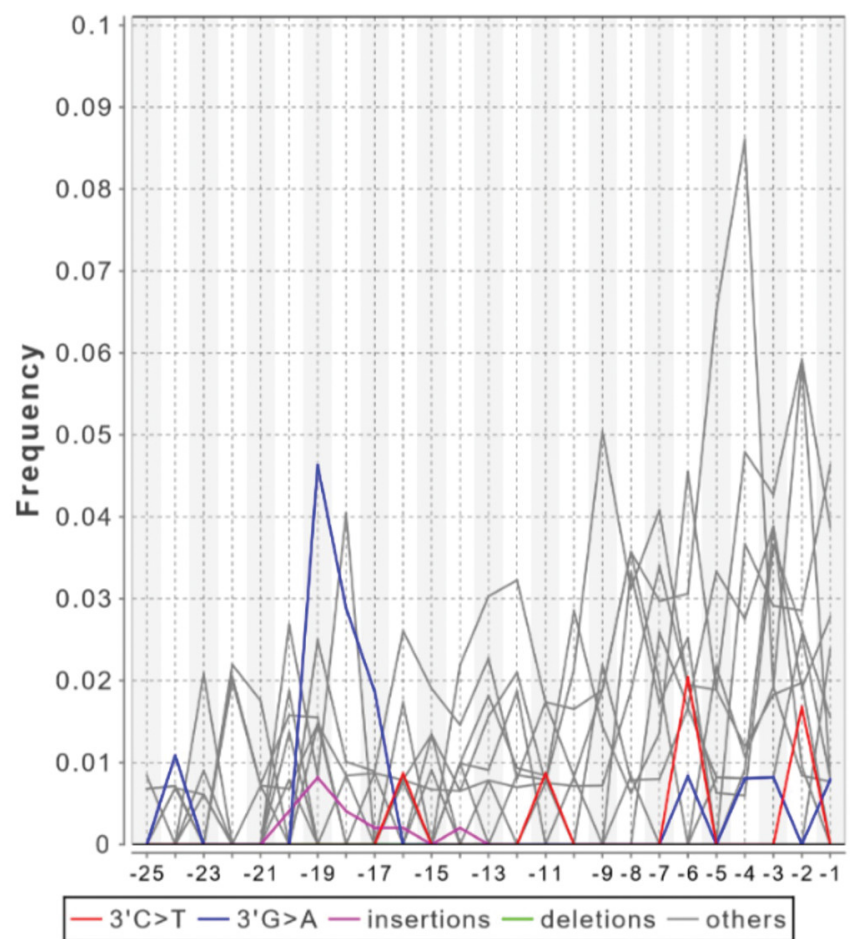

FIGURE 6.18 Damage pattern of 493 sequenced mitochondrial reads post-enrichment obtained from T.18 (zUM18B_oslo) sample with bleach pre-treatment and double digest method. Left: frequencies (in \%) of $\mathrm{C}$ to $\mathrm{T}$ misincorporations found at 5 ' end of sequences. Right: frequencies of $\mathrm{G}$ to A misincorporations found at 3 ' end of sequences PLOT PRODUCED BY MAPDAMAGE [GINOLHAC ET AL. 2O11]

5' end

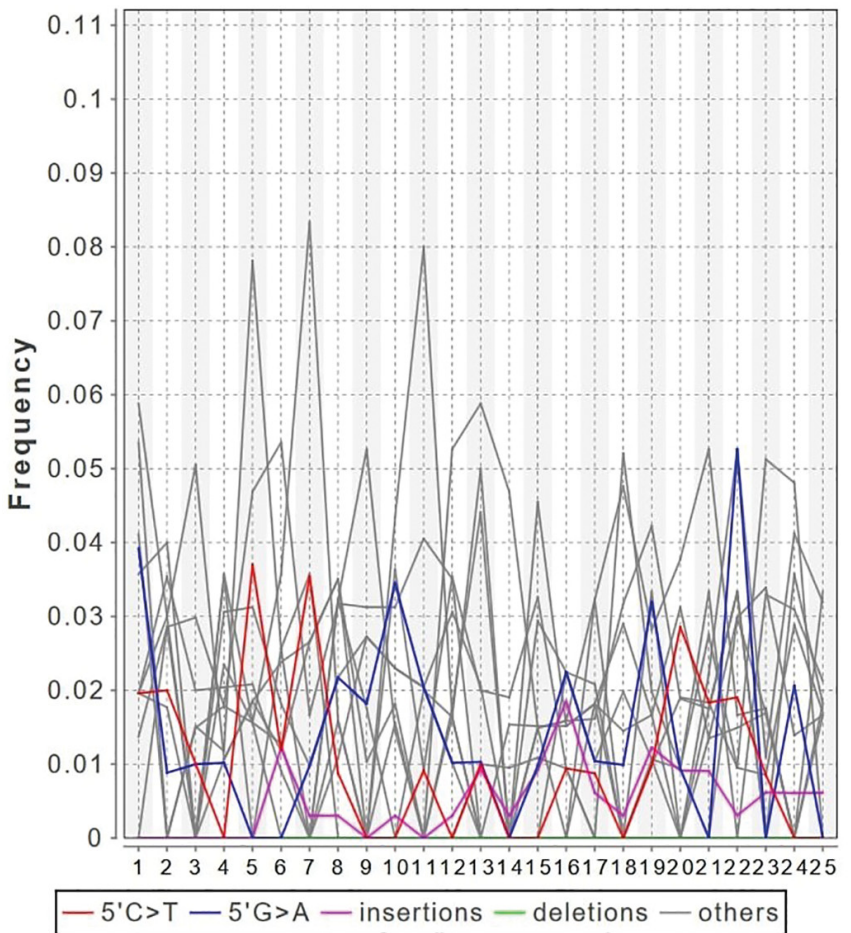

3' end

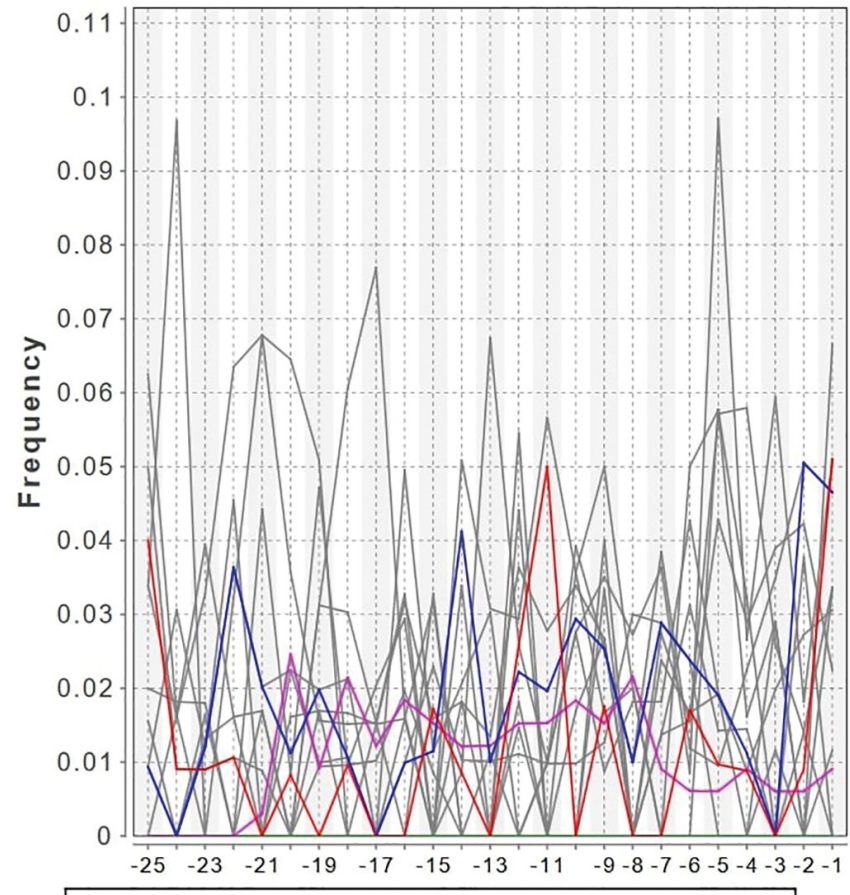

$-3^{\prime} \mathrm{C}>\mathrm{T}-3^{\prime} \mathrm{G}>\mathrm{A}$ - insertions - deletions - others

FIGURE 6.19 Damage plot for El-Zuma T.1o Individual, 333 shotgun reads used PLOT PRODUCED BY MAPDAMAGE [GINOLHAC ET AL. 2011] 

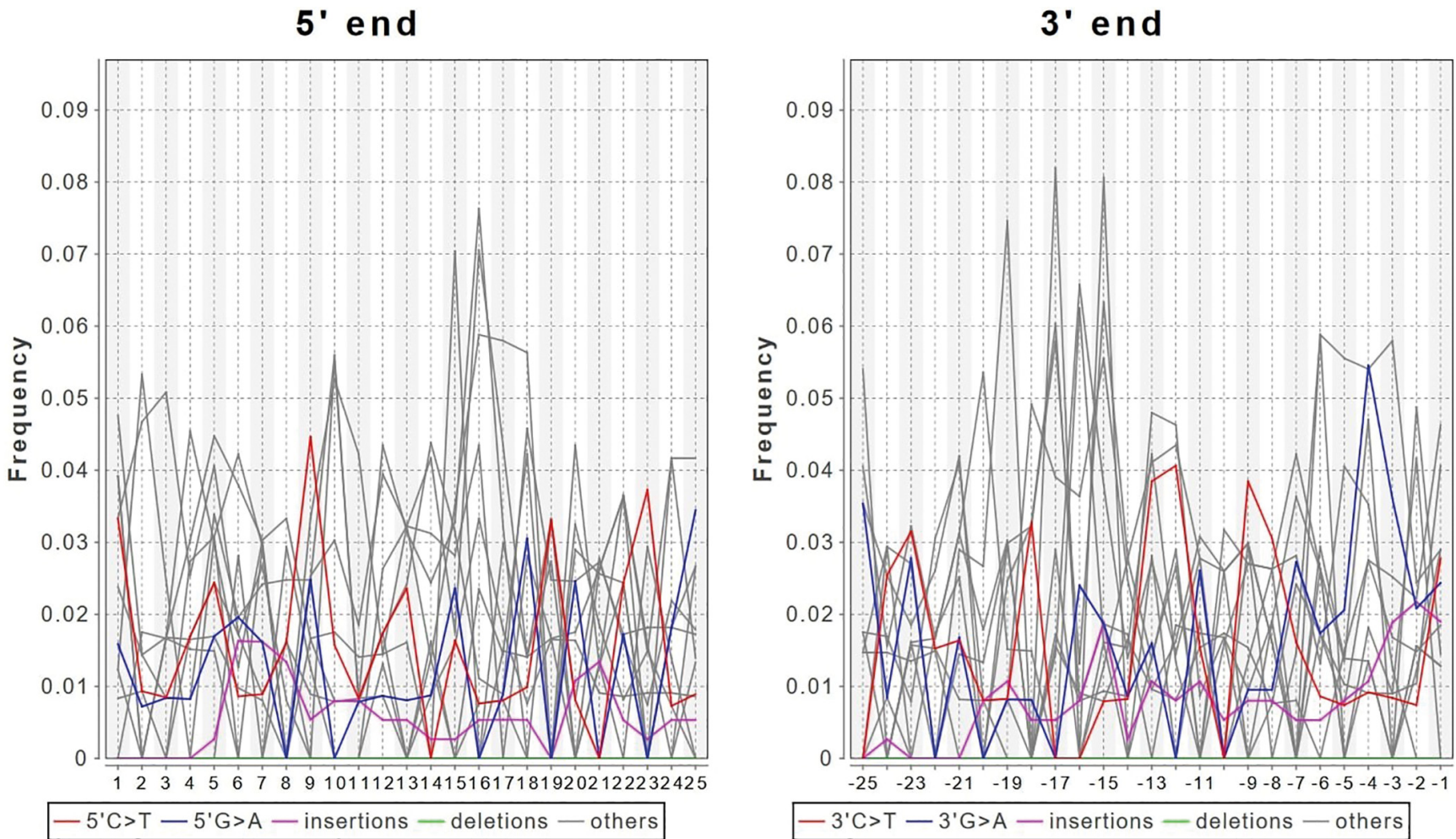

FIGURE 6.20 Damage plot for El-Zuma T.11 Individual, 378 shotgun reads used PLOT PRODUCED BY MAPDAMAGE [GINOLHAC ET AL. 2O11]
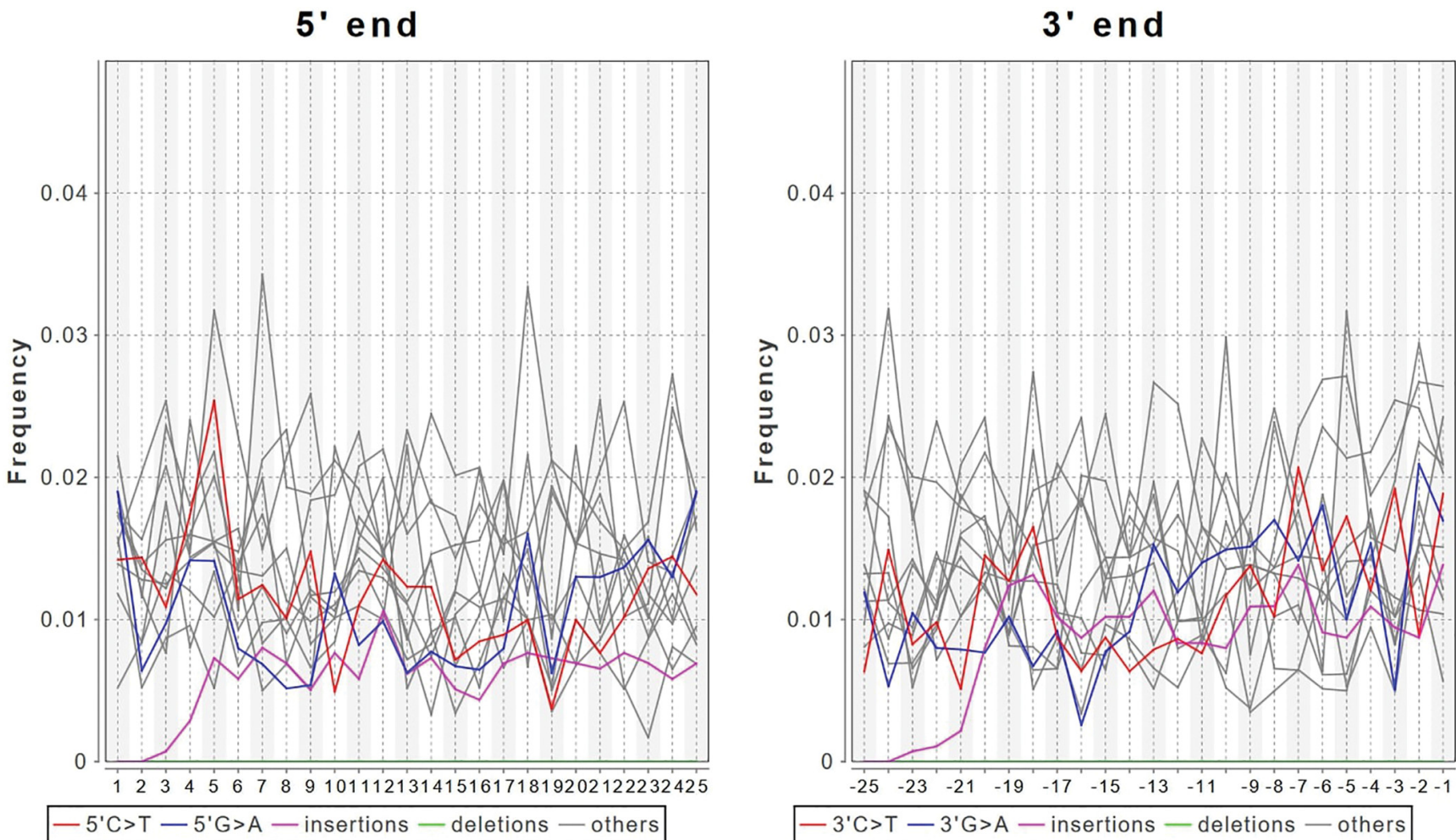

FIGURE 6.21 Damage plot for El-Zuma T.17 Individual, 2783 shotgun reads used PLOT PRODUCED BY MAPDAMAGE [GINOLHAC ET AL. 2O11] 
$5^{\prime}$ end

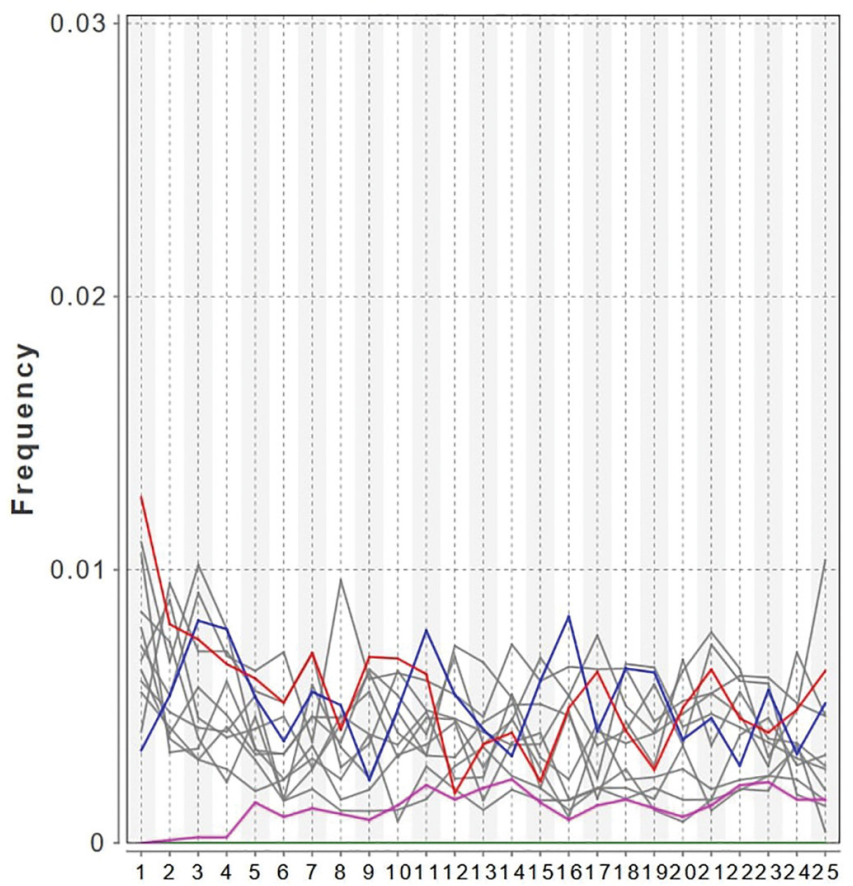

$5^{\prime} \mathrm{C}>\mathrm{T}-5^{\prime} \mathrm{G}>\mathrm{A}$ - insertions - deletions - others
3' end

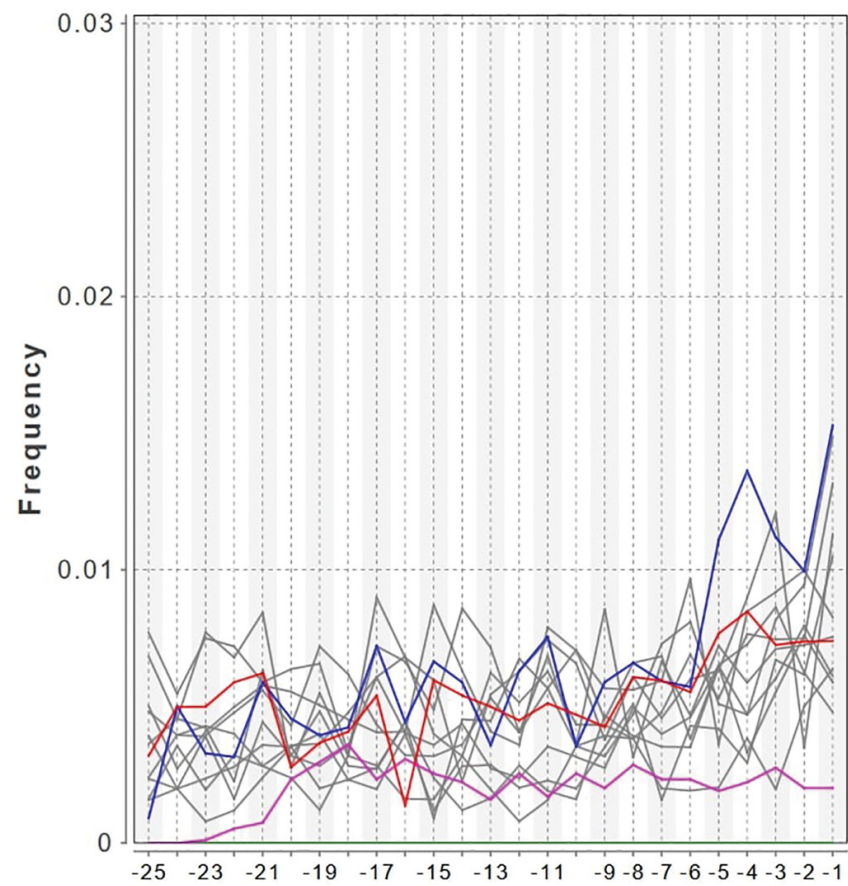

$3^{\prime} \mathrm{C}>\mathrm{T}-3^{\prime} \mathrm{G}>\mathrm{A}$ - insertions - deletions - others

FIGURE 6.22 Damage plot for El-Zuma T.18 Individual, 9477 shotgun reads used PLOT PRODUCED BY MAPDAMAGE [GINOLHAC ET AL. 2011]

5' end

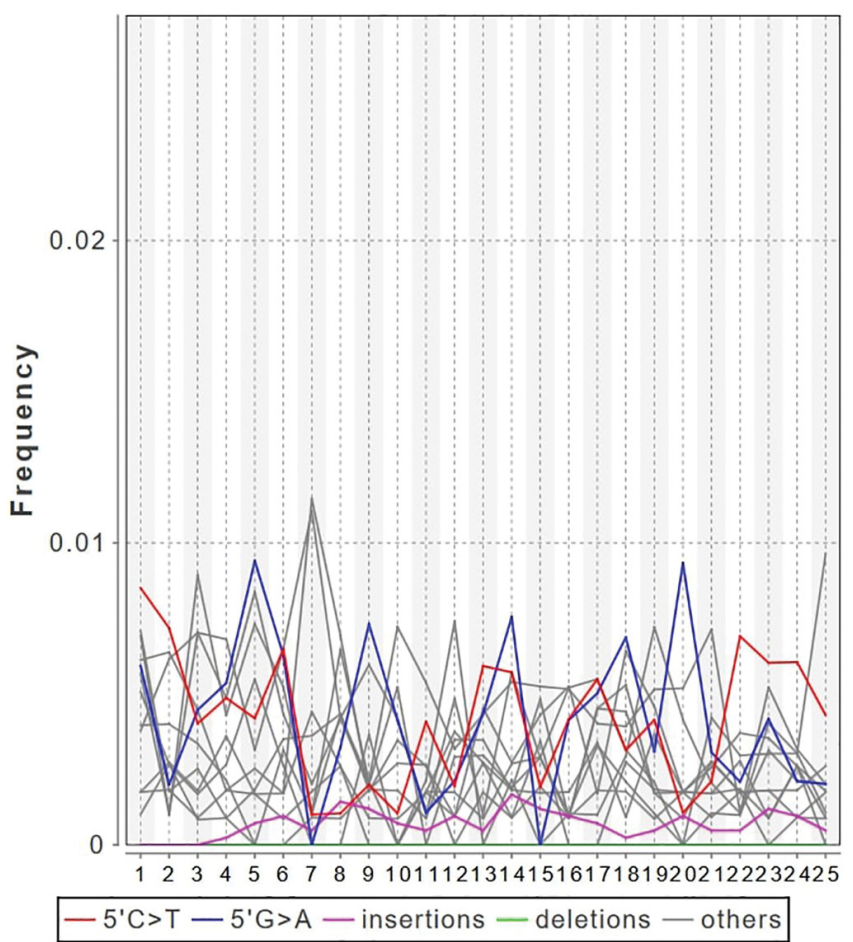

3' end

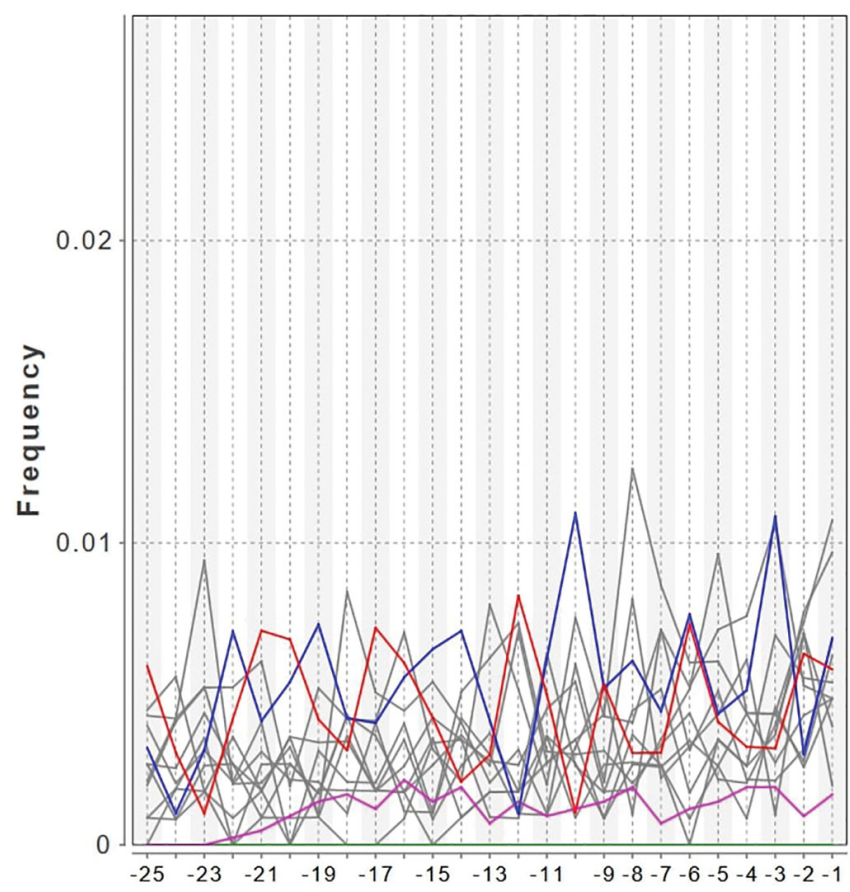

$-3^{\prime} \mathrm{C}>\mathrm{T}-3^{\prime} \mathrm{G}>\mathrm{A}$ - insertions - deletions - others

FIGURE 6.23 Damage plot for El-Zuma T.28 Individual, 4201 shotgun reads used PLOT PRODUCED BY MAPDAMAGE [GINOLHAC ET AL. 2011] 